\title{
TORUS: Theory of Reactions for Unstable iSotopes. Year 3 report.
}

I. J. Thompson, J. E. Escher, G. Arbanas, C. Elster, F. Nunes, A. Mukhamedzhanov

March 8, 2013 
This document was prepared as an account of work sponsored by an agency of the United States government. Neither the United States government nor Lawrence Livermore National Security, LLC, nor any of their employees makes any warranty, expressed or implied, or assumes any legal liability or responsibility for the accuracy, completeness, or usefulness of any information, apparatus, product, or process disclosed, or represents that its use would not infringe privately owned rights. Reference herein to any specific commercial product, process, or service by trade name, trademark, manufacturer, or otherwise does not necessarily constitute or imply its endorsement, recommendation, or favoring by the United States government or Lawrence Livermore National Security, LLC. The views and opinions of authors expressed herein do not necessarily state or reflect those of the United States government or Lawrence Livermore National Security, LLC, and shall not be used for advertising or product endorsement purposes.

This work performed under the auspices of the U.S. Department of Energy by Lawrence Livermore National Laboratory under Contract DE-AC52-07NA27344. 
Office of Science, U.S. Department of Energy

Office of Nuclear Physics

Nuclear Theory Division

\title{
TORUS:
}

\section{Theory of Reactions for Unstable iSotopes}

\author{
A Topical Collaboration for Nuclear Theory \\ Project Period: June 1, 2010 - May 31, 2015
}

Annual Continuation and Progress Report

Year-3: March 1, 2012 - February 28, 2013

Prepared by the TORUS Collaboration:

Goran Arbanas

Charlotte Elster

Jutta Escher

Akram Mukhamedzhanov

Filomena Nunes

Ian Thompson (Coordinating P.I.)

March 7, 2013 


\section{Contents}

$\begin{array}{llr}1 & \text { Introduction } & 3\end{array}$

2 Research $\quad 4$

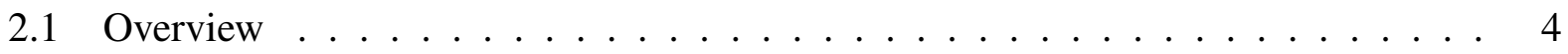

2.2 Coupled-channel Theory . . . . . . . . . . . . . . . . 4

2.2.1 Exploring the importance of Coulomb potentials with CDCC . . . . . 5

2.2.2 Distinguishing peripheral, surface, and interior contributions . . . . . . 6

2.2.3 Surface transfer operator . . . . . . . . . . . . . . 8

2.2.4 Connections to experiments and related efforts . . . . . . . . . . . 8

2.3 Faddeev Theory . . . . . . . . . . . . . . . . . . . 10

2.3.1 Coulomb potentials for generalized Faddeev AGS approaches . . . . . . . 10

2.3.2 Separable potentials for neutron scattering off closed shell nuclei . . . . . . 12

2.3.3 Generalized Faddeev AGS methods . . . . . . . . . . . . . . . . . . . . . 14

2.4 Capture Reactions . . . . . . . . . . . . . . . . . . . . . . 15

2.4.1 Semi-direct capture mechanisms . . . . . . . . . . . . . 15

2.4.2 Energy-averaging in statistical reaction models . . . . . . . . . . . . 17

2.4.3 Two-hole pair structure of the ${ }^{130}$ Sn ground state . . . . . . . . . . . . . 17

$2.4 .43 \alpha$ capture at low energies . . . . . . . . . . . . . . . . . 18

2.4 .5 Isobaric analogue resonances . . . . . . . . . . . . . . . . . 18

3 Project Management $\quad 19$

4 Postdoctoral Staff and External Collaborators $\quad 20$

5 Plans for Year $4 \quad 22$

6 Deliverables $\quad 23$

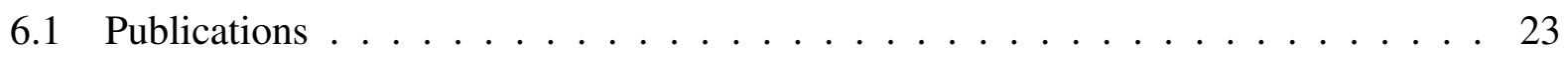

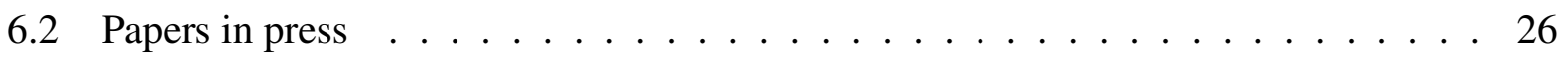

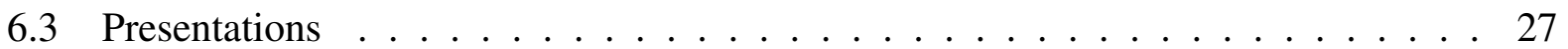

$\begin{array}{lr}\text { Bibliography } & 29\end{array}$ 


\section{Introduction}

\section{Background}

The TORUS collaboration derives its name from the research it focuses on, namely the Theory of Reactions for Unstable iSotopes. It is a Topical Collaboration in Nuclear Theory, and funded by the Nuclear Theory Division of the Office of Nuclear Physics in the Office of Science of the Department of Energy. The funding supports one postdoctoral researcher for the years 1 through 4 . The collaboration brings together as Principal Investigators a large fraction of the nuclear reaction theorists currently active within the USA.

\section{Mission}

The mission of the TORUS Topical Collaboration is to develop new methods that will advance nuclear reaction theory for unstable isotopes by using three-body techniques to improve directreaction calculations. This multi-institution collaborative effort is directly relevant to three areas of interest: the properties of nuclei far from stability; microscopic studies of nuclear input parameters for astrophysics, and microscopic nuclear reaction theory.

\section{Highlights from Year 3}

1. Publication of the detailed comparison of CDCC versus Faddeev [39].

2. Extraction of surface-term contributions to transfer cross sections - Section 2.2.2.

3. Numerous collaborations for analysis of experimental results - Section 2.2.4.

4. Separable potentials for neutron scattering on closed shell nuclei - Section 2.3.2.

5. Publication of new generalization of Faddeev-AGS equations with Coulomb distortion Section 2.3.3.

6. 16 papers published and another 4 submitted - Section 6 .

7. 18 presentations, including invited talks at various national and international venues, such as the HITES conference (June 2012), the DNP12 conference (October 2012) - Section 6.3. 


\section{Research}

\subsection{Overview}

We still need a general introduction that outlines very roughly the three sub-areas. This can then be followed by some details regarding the shift of focus/funding, as given just below.

New opportunities, arising from research completed earlier, motivated us to change priorities and to redirect funding from LLNL and ORNL to Ohio and Texas A\&M Universities for Year 3. This made it possible to support a student and to hire a postdoctoral researcher, who worked on the numerical implementation of the Faddeev-AGS equations earlier than the budgets would have otherwise allowed. For Year 4, we plan to return more closely to the original distribution of funds (minus the DOE-imposed funding reductions) between the Universities and the National Laboratories. One change, however, will be that the postdoc hired in Year 3 will now be hosted by Ohio University, instead of by MSU, as this provides the best match with the resources and expertise needed for the planned numerical work.

\begin{tabular}{lrr} 
Institution & Previous & Requested \\
\hline LLNL & $\$ 114 \mathrm{k}$ & $\$ 99 \mathrm{k}$ \\
MSU & $\$ 104 \mathrm{k}$ & $\$ 7 \mathrm{k}$ \\
TAMU & $\$ 27 \mathrm{k}$ & $\$ 24 \mathrm{k}$ \\
ORNL & $\$ 38 \mathrm{k}$ & $\$ 36 \mathrm{k}$ \\
Ohio & $\$ 11 \mathrm{k}$ & $\$ 101 \mathrm{k}$ \\
\hline Total & $\$ 294 \mathrm{k}$ & $\$ 267 \mathrm{k}$ \\
\hline
\end{tabular}

Table 1: Budget. Previous and requested plans for TORUS Year 4 (June 2013 - May 2014, incl.)

\subsection{Coupled-channel Theory}

A major motivation for our work originates from the new opportunities that rare isotope facilities offer. Theoretical work is required to both plan and interpret radioactive-beam experiments. The existing most advanced theoretical framework for carrying this out is coupled-channels theory, and work on this is reported in the subsections of $\S 2.2$.

An important and well-established implementation of coupled-channels theory is the continuum discretized coupled channel method (CDCC). We have in previous years completed careful comparisons of the CDCC method with exact three-body calculations within the Faddeev momentum space integral formalism, and identified specific shortcomings of the CDCC method (Section 2.2.1), as well as limitations of the present implementation of the Faddeev AGS equations.

It is also essential to identify what can and cannot be measured in experiments. We have investigated whether, and under which circumstances, transfer reactions probe the nuclear interior or the surface. We have studied bound as well as resonance final states (Section 2.2.2), as both types are important for extracting information that can be used to constrain nuclear structure models. 


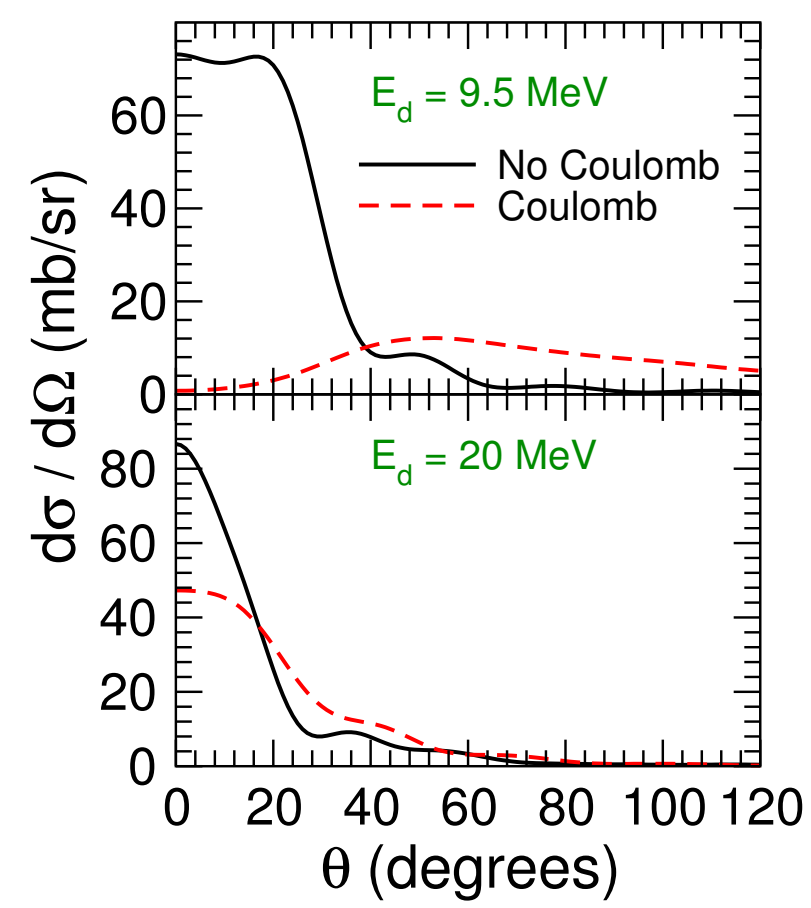

Figure 1: Transfer distributions for ${ }^{132} \mathrm{Sn}(\mathrm{d}, \mathrm{p}){ }^{133} \mathrm{Sn}$ reaction.

We have further explored the surface integral formalism, which was developed by A.M.M. in the first two years of the TORUS collaboration. This formalism makes use of concepts known from the successful and popular R-matrix theory. It paves the way for an alternative description of transfers to resonances, characterizes the measured cross sections in terms of resonance energies and partial widths, and can thus connect to astrophysically relevant capture reactions and nuclear structure studies. We have been testing various aspects of the approach and cast it in a form that makes it suitable for implementation within the reaction code FRESCO (Section 2.2.3).

We have also continued our efforts to connect with experimental efforts and related theory efforts (Section 2.2.4), and engaged the wider scientific community via workshops (Section 4).

\subsubsection{Exploring the importance of Coulomb potentials with CDCC}

\section{N. Upadhyay and F. Nunes}

The systematic comparison between the continuum discretized coupled channel method (CDCC) and the Faddeev AGS method (AGS) as implemented by the Lisbon group was the focus of the first 2 years of the MSU contribution to the TORUS collaboration. That work showed not only the lack of accuracy of CDCC for many (d,p) transfer and breakup observables, but also the limitation of the present implementation of the Faddeev AGS equations [6, 7]. This work is now published in Phys. Rev. C [39] and has received attention from the community, reflected in the number of invited talks on the subject.

Indeed, the reactions which can be calculated with the present implementation of Faddeev-AGS are very limited, particularly having in mind the reach of FRIB. For targets with charge $Z>20$, Coulomb effects require a larger screening radius and more partial waves; and this renders the solution of the AGS equations in the partial-wave representation as implemented in [6] impossible. 
In addition, the Padé summation technique used to solve the AGS equations converges slower at low energies $(10 \mathrm{MeV} / \mathrm{u})$, where couplings are strong, and it is not possible to obtain converged solutions for many cases of interest. It is exactly due to these limitations that TORUS needs to extend present techniques used in Faddeev-AGS and develop new methods to overcome those difficulties.

One particular challenge is the treatment of the long range Coulomb potentials. In the AGS formalism, this challenge manifests itself through singularities at momentum $p=0$. Apart from the Lisbon group, efforts in the past have approximated the Coulomb contribution with the assumption that, since $(\mathrm{d}, \mathrm{p})$ reactions involve light ions only (low $Z_{p} Z_{t}$ ), the transfer process was nuclear dominated and Coulomb would only provide a small correction to the cross sections around zero degrees scattering angle. While for light targets this is the case, as the charge increases we have found Coulomb effects can change the dynamics in a drastic way. In Fig.1 we show the angular distributions for ${ }^{132} \mathrm{Sn}(\mathrm{d}, \mathrm{p}){ }^{133} \mathrm{Sn}$ at two different beam energies. The solid black lines correspond to CDCC calculations neglecting Coulomb all together, and the dashed lines are the results including Coulomb. The same study was performed for other nuclei and the results are identical [28]. The conclusion of these studies is that, in $(\mathrm{d}, \mathrm{p})$ processes on intermediate/heavy mass targets, Coulomb effects are large and it is essential to include them correctly.

\subsubsection{Distinguishing peripheral, surface, and interior contributions}

\section{J.E. Escher, A.M. Mukhamdezhanov, and I.J. Thompson}

In order to identify which parts of the nucleus are probed by transfer reactions, we have examined the relative interior and exterior contributions of the reaction amplitude to calculated transfer cross sections. The reaction code FRESCO was modified in order to investigate separately the contributions from the internal and external parts of the reaction amplitude, where internal and external is defined in terms of the distance $r_{n A}$ between the transferred neutron and the target. DWBA calculations were carried out for a range of targets, ${ }^{12} \mathrm{C},{ }^{16} \mathrm{O},{ }^{20} \mathrm{O},{ }^{40} \mathrm{Ca},{ }^{48} \mathrm{Ca},{ }^{90} \mathrm{Zr}$, and ${ }^{208} \mathrm{~Pb}$, for incident deuteron energies between 11 and $82 \mathrm{MeV}$. Our main finding is that, although the post and prior DWBA amplitudes are equal, their behavior is quite different in the subspace over the variable $r_{n A}$. The prior formulation is clearly more sensitive to the nuclear interior (and thus to model assumptions about the interior structure), while contributions from the peripheral part of the $n+$ target system dominate the post form.

The surface integral formalism makes it possible to exploit this surface dominance. In this formalism, the transition amplitude is expressed as a sum of three terms: an interior prior term, an exterior post term, and a surface term: $M^{(D W B A)}=M_{\text {int }}^{(\text {post })}(0, a)+M_{\text {surf }}(a)+M_{\text {ext }}^{(\text {prior })}(a, \infty)$, with the notation $M(x, y)$ indicating the lower $(x)$ and upper $(y)$ limits of the integration over $r_{n A}$, and the surface term being evaluated at $r_{n A}=a$. This result was achieved [24] by separating the transition matrix element (in the post formalism) into an exterior and interior part, with respect to $r_{n A}$. Use of Green's theorem made it possible to convert the exterior part into a surface integral plus a remnant term ('prior' term). The interior post term is model-dependent, while the exterior prior and surface terms are related to the asymptotic properties of the wave function. The split into two asymptotic terms becomes significant when deuteron breakup effects are explicitly included via the continuum-discretized coupled-channels (CDCC) formalism [3]. In that context, the exterior 

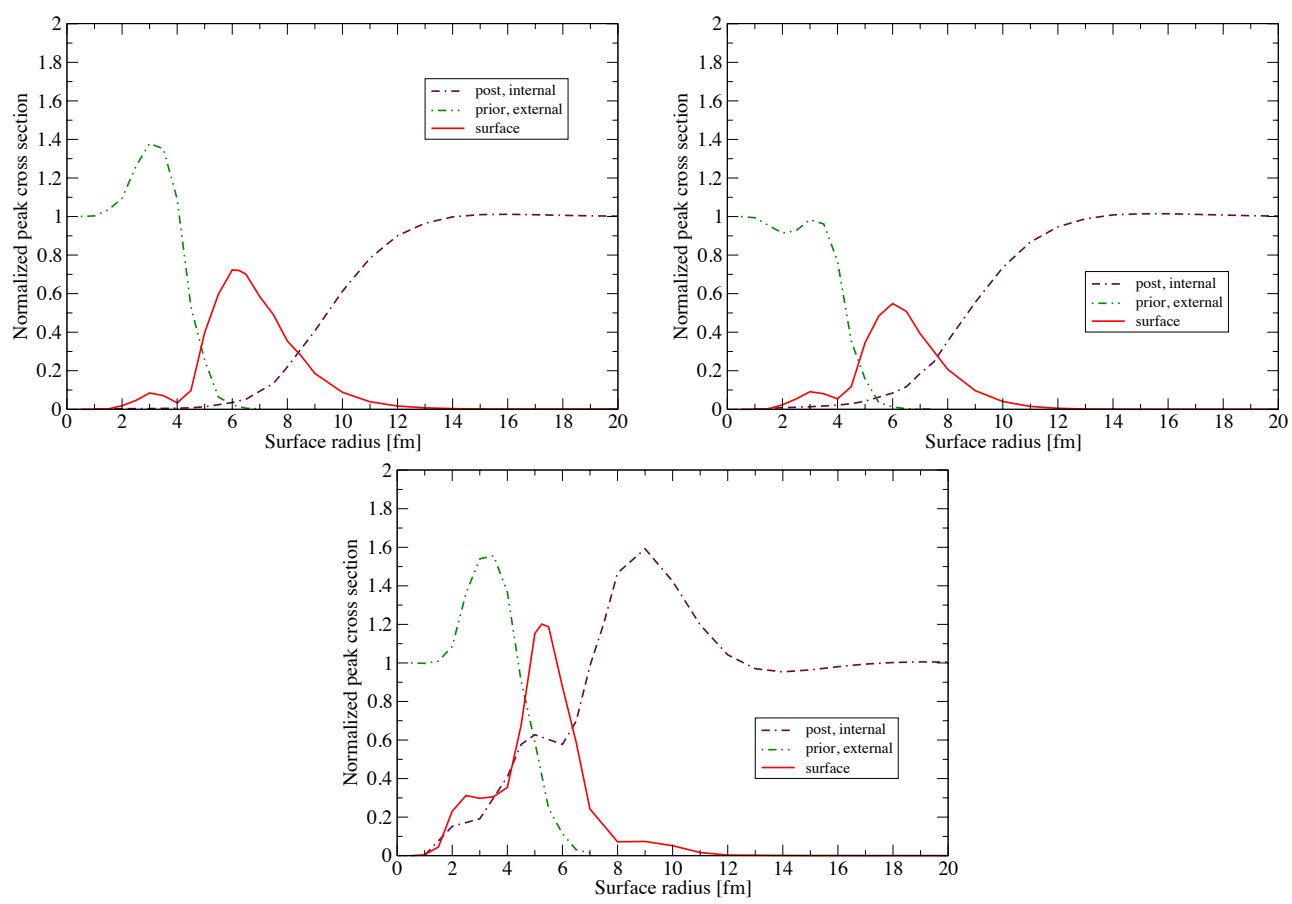

Figure 2: Interior, surface, and exterior contributions for ${ }^{48} \mathrm{Ca}(\mathrm{d}, \mathrm{p}){ }^{49} \mathrm{Ca}$ stripping to the $3 / 2^{-}$ground state, at $E_{d}=13$ (top left), 19.3 (top right), and $56 \mathrm{MeV}$ (bottom). Shown are the peak cross sections of the individual contributions (which are proportional to $\left|M_{\text {int }}^{(\text {post })}(0, a)\right|^{2},\left|M_{\text {surf }}(a)\right|^{2},\left|M_{\text {ext }}^{(\text {prior })}(a, \infty)\right|^{2}$, respectively), as a function of surface radius selected. The results are normalized to the peak cross section of the full calculation.

prior term no longer appears, i.e. $\quad M^{C D C C}=M_{\mathrm{int}}^{C D C C}(0, a)+M_{\mathrm{surf}}^{C D C C}(a)$.

Our analysis has so far been carried out in the DWBA formalism. While the surface contribution is found to be dominant at around 5-7 fm (see, e.g., Fig. 2 for the ${ }^{48} \mathrm{Ca}(\mathrm{d}, \mathrm{p}$ ) at three different beam energies), there are non-negligible contributions from both the interior (post) and exterior (prior) terms.

We have also carried out calculations that test the dominance of the surface term for transfer reactions that populate resonances. Experimentally, resonance structures are most often studied in elastic and inelastic scattering reactions. For those reactions, the phenomenological R-matrix approach has been extremely useful for the interpretation of experiments and for extracting resonance energies and widths from measured cross sections [22, 8]. The surface integral formalism [24] is essentially an extension of the R-matrix approach to $(\mathrm{d}, \mathrm{p})$ transfer reactions. It holds the potential to overcome present difficulties in describing transfers to resonance states and to become a practical and sound way for extracting structure information from transfer experiments, since: a) It reduces the dependence of the cross section calculations on the model used for the nuclear interior; $b$ ) it reduce (in DWBA) or eliminates (in CDCC) the impact of the slow convergence of calculations of the exterior term when resonances are considered; and c) it establishes a useful link between resonance properties and transfer observables, since the surface term $M_{\text {surf }}(a)$ can be parameterized in terms of quantities that are familiar from traditional R-matrix approaches. Since our resonance 
studies were carried out in the DBWA formalism, convergence was found to be difficult to achieve. The results obtained so far show trends similar to what was found for bound states, with reduced contributions from the nuclear interior.

An initial report on this work was published as a conference proceedings contribution and a longer paper will be submitted before the end of Year 3. We are currently working on implementing the extension to the CDCC approach (Section 2.2.3), which resolves the convergence issues.

Year 4 plan: We will carry out similar studies in the CDCC approach, using the tools described in Section 2.2.3 below, and investigate to what extent the combination of surface and breakup contributions can account for measured stripping cross sections.

\subsubsection{Surface transfer operator}

\section{A.M. Mukhamdezhanov, I.J. Thompson, and J.E. Escher}

In the previous section, we showed that for some transfer reactions we can choose a surface radius at which the surface transfer operator produces most of the transfer cross section. As a first approximation, therefore, we are going to calculate that surface term much more efficiently than in the above methods, and to treat the remaining terms as perturbations.

This surface term is defined at specific distance $r_{n A}=a$ of neutron-target distance, and depends only on the transfer wave function at that radius, and its derivative. Those properties of the wave function can be constructed from the R-matrix parameters for $n+A$ scattering, so the transfer cross sections can be directly fit in terms of the R-matrix parameters: the pole positions and their partial widths in each channel.

We have therefore formulated the matrix element elements of the surface operator, in the usual partial-wave basis used for transfers in Thompson's standard coupled-channels code FRESCO. These matrix elements are still finite-range operators, but, because of the surface constraint $a$, no angular integrals need to be performed numerically. The convergence of transfers to resonant states is expected to be extremely efficient, whether they are narrow or wide resonances.

Year 4 plan: We will calculate the matrix elements of the surface operator in the partial wave basis used by DWBA and CDCC calculations, to make a modified Fresco code. This will be tested for transfers to bound states and resonances, supplemented as necessary by interior-post and exterior-prior contributions. The first-order theory of transfers to resonances will be used to analyze existing experiments, and extracted partial widths will be compared to those found by other investigations. The effects of deuteron breakup in the initial channel will be considered, if time permits, using the CDCC approach. This work will be carried out by I.J. Thompson, J.E. Escher, and G. Arbanas.

\subsubsection{Connections to experiments and related efforts}

\section{Connecting existing methods with experiment}

\section{N. Upadhyay and F. Nunes in collaboration with A. Bey and K. Jones (UT)}

Recently, the ${ }^{10} \mathrm{Be}+\mathrm{d}$ reaction has been measured for a large range of energies [35] at the 
Holifield Radioactive Ion Beam Facility. A collaboration with experimentalists at the University of Tennessee was initiated with the intention of obtaining a comprehensive and consistent study of the elastic scattering, breakup and one-neutron transfer channels. Given the adequacy of the continuum discretized coupled channels (CDCC) method for the elastic and transfer channel, the analysis was performed in CDCC. The objectives of that work was i) to understand the role of breakup on elastic scattering and transfer for this weakly-bound system and ii) to unambiguously extract structure information for the bound states in ${ }^{11} \mathrm{Be}$ and quantify the errors in the extracted information. A publication of the results of this work is in preparation.

In addition, Nunes continues to connect with the experimental community. Below some of the efforts performed in 2012:

- Nunes was involved in preparing the proposal to use GRETINA at NSCL to study ${ }^{56} \mathrm{Ni}(\mathrm{d}, \mathrm{n}){ }^{57} \mathrm{Cu}$. This experiment was approved in the last NSCL PAC.

- Nunes was involved in preparing the proposal to use HELIOS at ANL to measure ${ }^{14} \mathrm{C}(\mathrm{d}, \mathrm{p}){ }^{15} \mathrm{C}$. This experiment was not approved in the last ANL PAC (priority was given to experiments that use CARIBOU).

- Nunes has been collaborating with Fred Sarazin from Colorado (and his former student Duane Smalley) on the analysis of TRIUMF data for the reaction ${ }^{12} \mathrm{C}\left({ }^{6} \mathrm{He},{ }^{4} \mathrm{He}\right){ }^{14} \mathrm{C}$.

\section{Integration of direct and compound reactions}

\section{J. Escher, in collaboration with experimentalists from U Richmond and LLNL}

The interplay of direct and compound mechanisms in one-nucleon transfer reactions is of interest to the TORUS collaboration. J. Escher has developed tools that provide information on the compound nucleus before decay by studying the statistical decay of the nucleus via gamma emission. This is important for testing theories that predict the direct and compound contributions in the reaction. These tools are being used (and refined) in collaboration with experimental colleagues, most recently by colleagues from the University of Richmond and with colleagues from LLNL. Measurements, carried out Lawrence Berkeley Laboratory, for $(p, d)$ and $(p, d)$ reactions on yttrium, gadolinium, and uranium nuclei, have resulted in three recent publications [34, 33, 19] and additional measurements were taken recently at the Texas A\&M Cyclotron Laboratory.

\section{Related efforts}

\section{F. Nunes and others at MSU}

The work of the TORUS postdoc, Neelam Upadhyay, in collaboration with various members of the collaboration, have been the central contribution of MSU to TORUS. However, there are other projects, that connect to the research developed in TORUS, and that provide leverage to TORUS. Below we provide a short list of those efforts:

- Luke Titus, an MSU PhD student, in collaboration with Nunes, have been investigating nucleon-nucleus non-local potentials. Titus has developed a code to solve the scattering 
problem with non-local interactions and we are now working with structure theorists to determine this interaction microscopically. Luke Titus is funded partly by NNSA and partly by NSF.

- Nunes, Capel and Esbensen have completed a detailed comparison of non-perturbative methods to describe the breakup of halo sytems [4]. These reactions are more challenging than deuteron induced reactions because the Coulomb interaction with the core is much stronger. But, as can be seen in [4], in some energy regimes there are non-perturbative methods much more efficient than the CDCC, that can still provide a good description of the process.

\subsection{Faddeev Theory}

\section{N. Upadhyay, V. Eremenko (postdocs), Ch. Elster, A. Mukhamedzhanov, and F. Nunes}

The implementation of this formalism will require the effort of various members of the collaboration. In early 2012, it was decided that:

- Ohio would focus on the optical potentials to be used in these AGS equations. We wanted to use separable interaction because these have the advantage of reducing the dimension of the integrations in the Faddeev kernel, transforming the AGS equations into a large set of one dimensional coupled integral equations. A separable form for the interactions is already assumed in the generalized AGS equations derived in [26]. The nucleon-nucleus interaction was the important missing piece, since there are several separable nucleon-nucleon interactions in the market. For more details, see section 2.3.2.

- TAMU would focus on investigating the formalism further, namely the application of the Coulomb regularization technique in the triangle diagrams for the rearrangement, inelastic and elastic channels. Also, the new PD at TAMU would initiate the coding of the generalized equations.

- MSU would numerically investigate the proposed method to handle Coulomb, with particular focus on the most challenging cases, namely heavy targets.

\subsubsection{Coulomb potentials for generalized Faddeev AGS approaches}

N. Upadhyay and F. Nunes, in collaboration with Ch. Elster, V. Eremenko and A. Mukhamedzhanov

By the summer of 2012, the formalism for a generalized Faddeev AGS approach had been derived by our collaboration [26], building on a separable representation for the interactions and including a new regularization technique to deal with the Coulomb correctly $[9,15]$. The regularization technique originally developed in the sixties, used a momentum-space Coulomb distorted wave representation $Z^{S C}\left(\mathbf{p}_{\beta}, \mathbf{p}_{\alpha}\right)$, defined by [26, 9]:

$$
Z^{S C}\left(\mathbf{p}_{\beta}, \mathbf{p}_{\alpha}\right)=\int \frac{d \mathbf{p}}{(2 \pi)^{3}} Z\left(\mathbf{p}_{\beta}, \mathbf{p}\right) \psi_{\mathbf{p}_{\alpha}}^{C}(\mathbf{p}) .
$$

Here the Coulomb wave function $\psi_{\mathbf{p}_{\alpha}}^{C}(\mathbf{p})$ is defined at (initial) momentum $\mathbf{p}_{\alpha}$ and $Z\left(\mathbf{p}_{\beta}, \mathbf{p}\right)$ is the half-off-shell effective potential with the on-shell final state momentum, $\mathbf{p}_{\beta}$. 

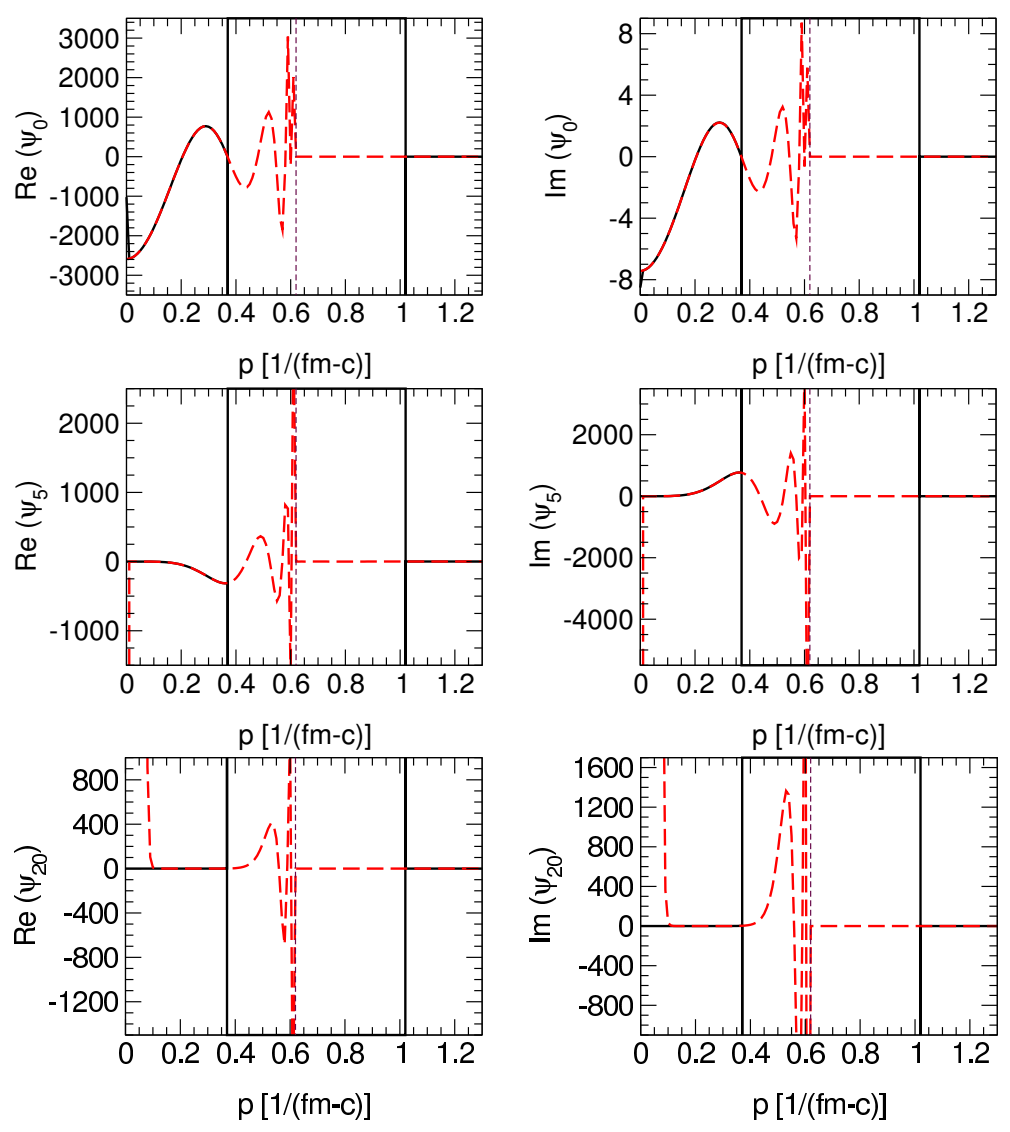

Figure 3: Coulomb wave functions for $\mathrm{p}+{ }^{208} \mathrm{~Pb}$ at $\mathrm{E}_{\mathrm{cm}}=8 \mathrm{MeV}: l=0,5$, and 20.

Given that the generalized AGS equations [26] are written using the momentum-space Coulomb distorted wave representation, the implementation needed to begin with a perfect understanding of Eq. (1). In the summer of 2012, the PD at MSU, with guidance from TAMU, has rederived the formalism in [26], with particular focus on the partial wave decomposition of the momentum-space Coulomb wave function. A detailed analysis of the Coulomb wave function in momentum space was performed, and its dependence on angular momentum (this studied relies on a close comparison of the results obtained with a newly developed fortran90 code and those from mathematica). In Fig.3 we show the dependence of the Coulomb function in momentum, after partial wave decomposition. Due to the large charge of the system, in addition to the known poles at $p=0$ and $p=p_{\alpha}$, variable oscillations appear which require some care. We also performed similar studies for the relevant integrand (obtained after partial wave decomposition of Eq. (1)). At present, a number of numerical integration methods are being explored to handle the difficulties involved in calculating these integrals to the desired accuracy. Elster and Nunes have worked in close collaboration with the PD at MSU in all matters pertaining the numerical implementation.

While these initial studies assumed a Yamaguchi form for the interaction [26]: $Z\left(\mathbf{p}_{\beta}, \mathbf{p}\right)=$ $-2 \mu_{12} Z_{1} Z_{2} e^{2} /\left[\left(\mathbf{p}_{\beta}-\mathbf{p}\right)^{2}+\mathfrak{K}^{2}\right]$, to allow comparisons with analytic results, it is clear that our implementation needs to be more flexible. We want to incorporate the separable form factors provided by the EST method (see next section), which are in numerical form. Part of the latest work at MSU has been the generalization of the new Coulomb distorted effective potential code to 
be able to cope with an arbitrary numerical form for the interaction. A full report of the PD efforts on this topic is available upon request [38].

Plans for Year 3 Q4: The appointment of the TORUS PD at MSU has an end date of 15th July 2013. From now until then, the TORUS efforts at MSU will continue to focus on the treatment and practical implementation of the momentum-space Coulomb distorted wave representation, used in the AGS equations of [26].

Plan for year 4: For the concluding period of TORUS, Nunes will continue to work closely with collaborators at OU in the implementation of the AGS equations, the primary focus of the remainder of the TORUS work. Given the extended experience of Deltuva in implementing AGS equations, Nunes will continue to collaborate with Deltuva, to bring his invaluable knowhow to our collaboration.

\subsubsection{Separable potentials for neutron scattering off closed shell nuclei}

\section{Ch. Elster and L. Hlophe}

For the generalized AGS equations adapted to calculate (d,p) reactions for arbitrary nuclei, we proposed to use separabilized potentials between the constituents of the reaction. For nuclear reactions this means the nucleon-nucleon interaction for the deuteron subsystem and the neutron or proton optical potentials between the neutron and proton in the deuteron and the target nucleus. A separable nucleon-carbon-12 optical potential was proposed in Ref. [23], consisting of a rank-1 Yamaguchi-type form factor fitted to the positive energies and a similar term describing the bound states in the nucleon-carbon-12 configuration. Considering the variety of nuclei for which there is or will be experimental information available from $(\mathrm{d}, \mathrm{p})$ reactions, one will need a separabilization procedure that is sufficiently general so that it can be applied to a variety of nucleon-nucleus optical potentials over a wide range of nuclei. In addition, transition amplitudes calculated from phenomenological optical potentials need to be well represented over a wider range of energies.

The separable representation of two-body interactions suggested by Ernst-Shakin-Thaler [13, 12] (EST) looks well suited to achieve this goal. Though the EST scheme was extensively used to represent NN t-matrices [18, 17], it has to our knowledge not yet been applied to optical potentials for nucleon-nucleus scattering. We first concentrated on developing a separable representation of neutron-nucleus optical potentials. For ease of comparison with existing calculations from FRESCO, we based our development work on the the Chapel-Hill CH89 phenomenological global optical potential [40].

We started our study of applying the EST scheme to represent the partial wave projected tmatrices for neutron scattering from ${ }^{48} \mathrm{Ca}$ based on the Chapel-Hill $\mathrm{CH} 89$ phenomenological global optical potential [40] between 0 and $50 \mathrm{MeV}$ c.m. energy. This is the energy regime relevant to the planned $(\mathrm{d}, \mathrm{p})$ calculations. It turned out that with already a rank-3 separable potential a quite reasonable description of the partial wave s-matrix elements can be obtained, as is shown in Fig. 4 for the $l=1$ partial partial wave s-matrix.

The work on the separabilization of the $n+{ }^{48} \mathrm{Ca}$ optical potential allowed us to conclude that it is sufficient to find a good representation of the central term of the optical potential. Since the spin-orbit term is relatively small compared to the central term, the EST support points determined for the central part will also give a good description of the entire optical potential, i.e. central plus 

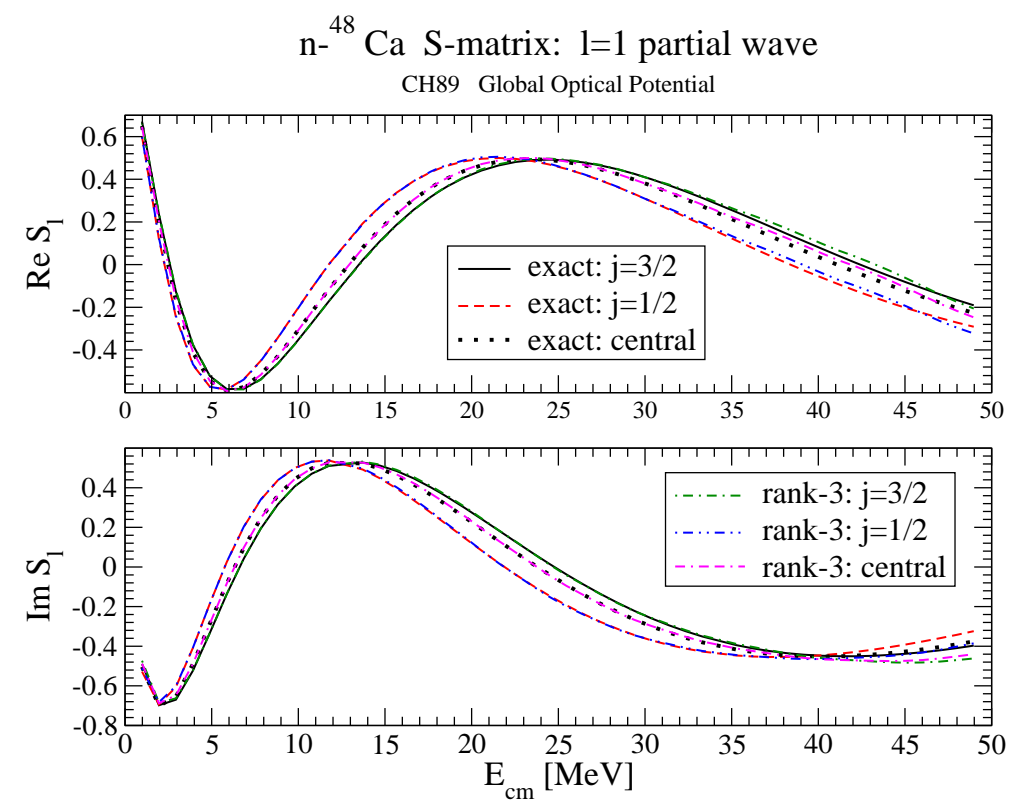

Figure 4: The $l=1$ partial wave s-matrices of the CH89 optical potential calculated exactly and compared to rank-3 separable representations.

spin-orbit term. This is a very useful result, since the amount of amplitudes to fit is cut into half. In addition, the lower partial wave S-matrices need the highest rank separable potential for being well represented. Thus, once the EST support points for the low $l$ partial waves are determined, they can be safely applied to all higher partial waves.

In order to test our implementation of the EST scheme, we also separabilized the $n+{ }^{132} \mathrm{Sn}$ and $\mathrm{n}+{ }^{208} \mathrm{~Pb}$ Chapel-Hill $\mathrm{CH} 89$ global optical potential parameterizations [40]. As the nuclei become heavier, the partial wave S-matrices acquire more structure. This means that one needs a higher rank separable potential for an adequate description of especially the lower $l$ partial waves. For the heaviest nucleus, ${ }^{208} \mathrm{~Pb}$, a rank-6 parameterization is required for a good description of the lower $l$ partial waves S-matrices in the energy range up to $50 \mathrm{MeV}$ c.m. energy. Obviously, for much higher angular momenta, a lower rank will suffice. We tested, that once EST support points have been determined to give a good description of the $n+{ }^{208} \mathrm{~Pb}$ optical potential, then, using the same points, the optical potentials for $n+{ }^{132} \mathrm{Sn}$ and $n+{ }^{48} \mathrm{Ca}$ are well described also. Thus, for an application where the size of the rank is not relevant, one can have a "universal" set of support points. Using lower rank representations is always possible when e.g. using the optical potential in a more restricted energy range, or using a lower rank for lighter nuclei. Our finding will be prepared for a manuscript for publication within the next two months.

Year 3 and 4 plans: In order to have an alternative, more recent global optical potential fit available for future calculations, we chose to add the global fit of Ref. [41] to our repertoire. Very specifically, in order to start the work on the AGS equations, postdoctoral researcher Vasily Eremenko visits Ohio University during the month of February. We are starting from old AGS code used to compute deuteron elastic scattering and processes off ${ }^{12} \mathrm{C}$ as a three-body problem [1]. Since we need to work on all aspects on the problem, we want to use the results of the old code 
for specific benchmarks. The work of Ref. [1] uses a very simple rank-1 optical potential. To create a general code, we need to incorporate the work on separable optical potentials into a new code. After careful consideration with S.P. Weppner, we came to the conclusion that his global optical potential provides the best description of ${ }^{12} \mathrm{C}$ elastic scattering data, and well suited to isolate the nucleon-nucleus interaction piece of the code. We believe it is most efficient to dissect the existing code and replace the different pieces while creating a new code, so that we can keep checkpoints in phases of the work.

The separabilization techniques described above are at present carried out for n+nucleus optical potentials. In order to describe $p+$ nucleus optical potentials one needs to calculate the Coulomb distorted t-matrix elements, and refit with a Coulomb term included [41]. The calculation of Coulomb distorted matrix elements has been a major focus of the post doctoral researcher Neelam Upadhyay at MSU, as described in the previous section. Eventually our separable optical potentials will serve as test cases for her procedure of calculating Coulomb distorted matrix elements.

This work will be continued through the end of the 3rd year funding period and is supported in part by TORUS and in part by DOE contract No. DE-FG02-93ER40756. The stipend of the graduate student Linda Hlophe has been split between the two grants. After completing his work on the TORUS grant, Linda Hlophe will most likely continue his work on optical potentials, but now on a more fundamental level working on microscopic descriptions.

A further purpose of the visit of postdoctoral research Vasily Eremenko to Ohio University is to determine if he is suited to continue on the AGS project, but at Ohio University rather than at Texas A\&M university.

\subsubsection{Generalized Faddeev AGS methods}

\section{Eremenko (postdoc) and A. Mukhamedzhanov}

The original Faddeev equations are written for $3 \rightarrow 3$ collisions. To apply them for the deuteron stripping it is convenient to rewrite them in the AGS form, in which the Faddeev equations are written directly for coupled reaction amplitudes for $2 \rightarrow 2$ binary processes. These AGS equations are used as a starting point. However, these equations are not enough to take into account the whole complexity of the deuteron stripping reactions, because in the Faddeev approach the target is treated as a structureless constituent particle.

In year 2 of our collaboration, A.M. Mukhamedzhanov had developed a new formulation of the generalized Faddeev equations in the AGS form for the deuteron stripping, which includes explicitly the Coulomb interactions and target excitations. The Coulomb interaction in the AGS approach appears in the three-ray vertex form factors in the effective potentials and in the four-ray vertex in the triangular diagrams. Applying the two-potential equation allows one to remove the non-compact singularity in the triangular diagram describing the elastic scattering and containing the $\mathrm{p}-A$ Coulomb scattering amplitude. The AGS equations can also be rewritten in the Coulomb distorted wave representation, in which the reaction amplitudes and the effective potentials are sandwiched by the Coulomb distorted waves in the initial and final states. Another important topic of our research is the investigation of the singularities of the integrals containing the off-shell Coulomb scattering amplitude. This is necessary to prove the compactness of the Faddeev equations. The practical application of the obtained equations requires regularization of the integrals 
containing the off-shell Coulomb scattering amplitudes. Note that in a standard procedure involving the screening of the Coulomb potential, it is tacitly assumed that, in the limit of the screening radius $R \rightarrow \infty$, all the integrals containing the Coulomb scattering amplitude have well-defined limits and that the Coulomb screening affects only the Coulomb distorted waves in the initial and final states.

In the $\mathrm{d}+A$ case for deuteron scattering under consideration, when only two particles are charged, only one off-shell Coulomb scattering amplitude of the $\mathrm{p}-A$ scattering is needed. It is shown how to deal with all the integrals containing the Coulomb off-shell T-matrix. Applying our regularization procedure, we obtain the expression for the effective potentials in the AGS equations which are free of the singularities caused by the Coulomb distortions in the initial and final states. It is shown that the Coulomb-modified form factors in the transfer amplitudes and in the triangular diagrams do not contain non-integrable singularities. The target excitation is taken into account following the formalism developed by projecting space on the three-body one. The final generalized AGS matrix equations are written in the form which includes explicitly Coulomb interactions, target excitations and spins of the particles. The work was published as [26].

Because the solution of the AGS equations is greatly simplified for separable potentials, we use a separable potential approach, assuming that these approximate the realistic $N N$ potentials and $N A$ optical potentials. The important code to represent these potentials in a separable form is being developed at Ohio (see Section 2.3.2). These will later be generalized to include target excitation, and enable us to go beyond simple models of three inert bodies.

\subsection{Capture Reactions}

\section{G. Arbanas, I.J. Thompson, and J. Escher}

\subsubsection{Semi-direct capture mechanisms}

We completed the computation of direct-semidirect (DSD) capture ${ }^{130} \mathrm{Sn}(\mathrm{n}, \gamma)$ and the results shown in Fig. 5 were published in [20], thus reducing the uncertainty of the computed direct capture (by various models shown in this figure) by orders of magnitude. Besides the DSD capture, we attempted to address the applicability of the compound nuclear (i.e. statistical) capture computations, by using various statistical models implemented in TALYS, since the input to the nucleosynthesis models requires a total capture cross section that is a combination of the DSD and compound captures. Furthermore, nucleosynthesis models tend to be especially sensitive to the total captured cross section near doubly-closed shell nuclei like ${ }^{132} \mathrm{Sn}$. This motivated further measurements of (d,p) [5] on a stable ${ }^{124} \mathrm{Sn}$ and unstable ${ }^{126,128} \mathrm{Sn}$ isotopes (measured in inverse kinematics) that could lead to better computations of direct-semidirect and compound neutron capture cross sections.

An attempt to explain a surprisingly low level-density around the neutron threshold of ${ }^{131} \mathrm{Sn}$ was made using the relativistic mean field theory in [43]. The relativistic mean field theory computation yielded bound single-particle states of ${ }^{131} \mathrm{Sn}$ and ${ }^{133} \mathrm{Sn}$ that are in reasonable agreement with the $(\mathrm{d}, \mathrm{p})$ data and it also predicted single-particle resonances in the continuum.

Although all extant statistical models for computation of Hauser-Feshbach cross-section from 


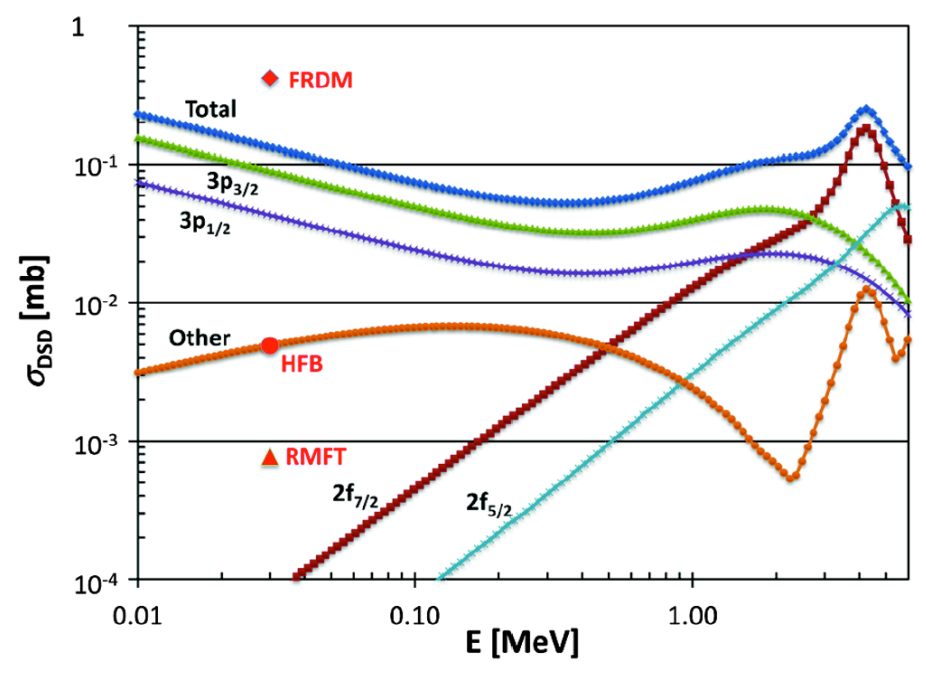

Figure 5: Computation of direct-semidirect neutron capture cross section for the ${ }^{130} \operatorname{Sn}(\mathrm{n}, \gamma)$ reaction reported in [20]. Shown for comparison (single points) are the calculations for $30 \mathrm{keV}$ neutrons using the finite range droplet (FRDM), the Hartree- Fock-Bogoliubov (HFB), and relativistic mean field theory (RMFT) models. Of these, only the FRDM predicted both the $3 \mathrm{p}_{3 / 2}$ and $3 \mathrm{p}_{1 / 2}$ single-neutron states to be bound.

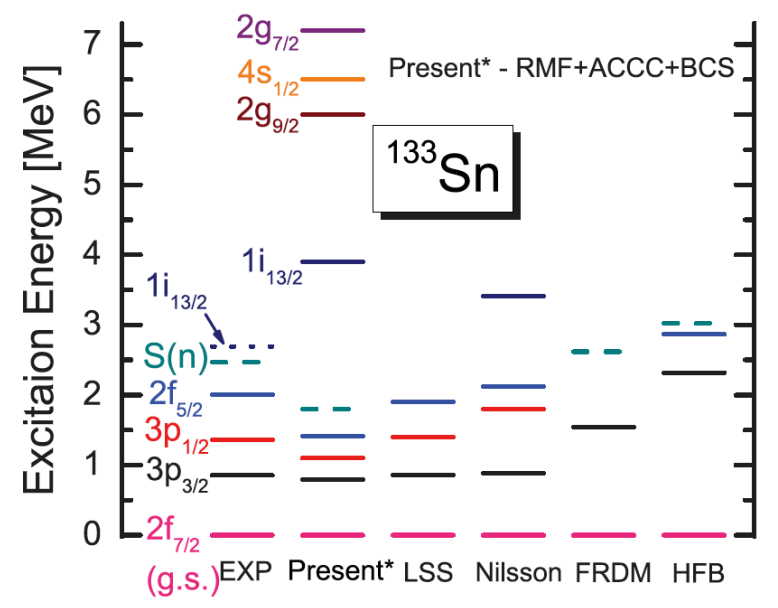

Figure 6: Bound levels and resonant levels in ${ }^{133}$ Sn calculated by our approach with the NL3 effective interaction compared with the latest data and calculations from the LSS, Nilsson, FRDM, and HFB models. The available neutron capture thresholds $\mathrm{S}(\mathrm{n})$ are also shown.

TALYS yield a compound capture that is significantly larger than the DSD capture, such models may not be reliable for reactions for which the density of compound nuclear states is too low, and this may turn out to be the case here, as was argued in [43]: "Our RMF + ACCC + BCS successfully reproduces observed single-particle bound levels in ${ }^{131,133} \mathrm{Sn}$ and self-consistently predicts single-particle resonant levels with densities too low for widely used traditional statistical model treatments of neutron capture cross sections on ${ }^{130,132}$ Sn employing Fermi gas level density formulations", as plotted in Fig. 6. On the other hand, if the models in TALYS turn out to be applicable to ${ }^{130} \operatorname{Sn}(\mathrm{n}, \gamma)$ it would be an indication that the compound capture may be the dominant capture process and thus greater than the DSD capture.

It is conceivable the low energy-resolution of the experimental data for ${ }^{130} \mathrm{Sn}(\mathrm{d}, \mathrm{p})$ in inverse 
kinematics, from which the level-density of states in ${ }^{131} \mathrm{Sn}$ was inferred, may not have been sufficiently high to resolve underlying fragmentation of single particle levels. Such fragmentation of single-particle levels, would imply that the statistical (i.e. the compound nuclear) capture contribution to the total capture would be increased due to the fragmentation. A (particle) + (collective core) model that was used to successfully explain the fragmentation of single particle states of ${ }^{207} \mathrm{~Pb}$ may be used as circumstantial evidence that a similar fragmentation of single particle levels would occur in ${ }^{131} \mathrm{Sn}$.

Presently we are continuing this work in collaboration with the ORNL experimentalists in order to compute direct-semidirect capture cross section on ${ }^{80} \mathrm{Ge}$, for which ${ }^{80} \mathrm{Ge}(\mathrm{d}$, p) data was recently measured. ${ }^{80} \mathrm{Ge}$ is two neutrons short of the neutron closed shell $(N=50)$ to ${ }^{82} \mathrm{Ge}$ and therefore its capture cross section may influence nucleosynthesis models.

\subsubsection{Energy-averaging in statistical reaction models}

Work on energy-averaging has not been performed this year, because of a changes of TORUS priorities.

\subsubsection{Two-hole pair structure of the ${ }^{130}$ Sn ground state}

We generalized the exact solution to the pairing Hamiltonian with constant interaction strength [32] to a state-dependent interaction strength ${ }^{1}: H=\sum_{i} \varepsilon_{i}\left(a_{i_{+}}^{\dagger} a_{i_{+}}+a_{i_{-}}^{\dagger} a_{i_{-}}\right)-g \sum_{i} \sum_{j} c_{i} c_{j} a_{i_{+}}^{\dagger} a_{i_{-}}^{\dagger} a_{j_{+}} a_{j_{-}}$, where the $\varepsilon_{i}$ is the energy of the $i$-th single-particle state, $c_{i}$ are properly normalized separable interaction coefficients, and $g$ is an overall coupling strength. The derivation of Richardson and Sherman in [32] can be retraced to yield a generalized analytical solution for the $N$-pair wave function:

$$
\left|\Psi_{N}\right\rangle=\prod_{\beta=1}^{N} \sum_{i} \frac{c_{i}}{2 \varepsilon_{i}-E_{\beta}} a_{i+}^{\dagger} a_{i_{-}}^{\dagger}|0\rangle
$$

where pair energies $E_{\beta},(\beta=1, \ldots, N)$ are solutions to a generalized set of coupled non-linear equations that retain the form of the original Richardson's model:

$$
\frac{1}{2} \sum_{i} \frac{c_{i}^{2}}{2 \varepsilon_{i}-E_{\beta}}=\frac{1}{2 g}+\sum_{\beta^{\prime}(\neq \beta)} \frac{c_{\beta^{\prime}}^{2}}{E_{\beta^{\prime}}-E_{\beta}} \quad i=1 \ldots N
$$

The total eigen-energy of this pairing Hamiltonian is then $E=\sum_{i} \varepsilon_{i} \nu_{i}+\sum_{\beta} E_{\beta}$,where the pair energies $E_{\beta}$ are solutions to Eq. (3), and where $\nu_{i}$ is a seniority of the $i$-th single-particle state, which for non-degenerate single-particle states is 0 or 1 . Since the number of variational parameters in this model equals the number of single particle levels, this variational model ought to describe the excitation spectrum of ${ }^{130} \mathrm{Sn}$ (or other nuclei) more accurately than a constant interaction strength Richardson model does. The pairing mechanism is essential to describe the ground state of ${ }^{130} \mathrm{Sn}$, or other even-mass tin isotopes, just as it successfully described the ground state of even-mass lead isotopes in [32].

\footnotetext{
${ }^{1}$ Setting $c_{i}=1$ in this section recovers the special case of the original exactly solvable Richardson's model.
} 


\subsection{4 $3 \alpha$ capture at low energies}

\section{N. Nguyen, F. Nunes and I.J. Thompson}

F. Nunes and her student Ngoc Nguyen (with others) have worked on Faddeev equations for the triple-alpha scattering and the triple-alpha reaction. Again, the challenge in this work was the long-range Coulomb interaction. A combination of the R-matrix method, R-matrix propagation, and screening, was used to solve the problem. Results are published in [27]. Ngoc Nguyen is funded partly by NNSA and partly by NSF.

\subsubsection{Isobaric analogue resonances}

\section{G. Arbanas and I.J. Thompson}

With our implementation within our coupled-channels code FRESCO of the main Lane coupling term [2], we have described the charge-exchange interaction that transforms an incident proton into a neutron, and gives resonances in elastic scattering when the neutron sub-threshold energy is near an unoccupied single-particle state. We see doorway resonances when the neutron energy is near a bound state. At the same time, a target neutron must have changed to a proton, so it must have been in an occupied neutron state with quantum numbers such that a proton with those parameters is not Pauli blocked. We therefore extended the Lane coupled-channels formalism to follow the non-orthogonality of this neutron channel with that configuration of an inelastic outgoing proton, and the target being left in a particle-hole excited state. This has been tested

for ${ }^{208} \mathrm{~Pb}$, for which good $\left(\mathrm{p}, \mathrm{p}^{\prime} \gamma\right)$ coincidence data exists [31], and we will soon make predictions for the equivalent processes for ${ }^{132} \mathrm{Sn}$, and talk to experimentalists about possible experiments. Experiments such as [36] show the methods are also useful for light nuclei.

Plan for Year 4: We will complete testing of our treatment of isobar-analogue resonances, including multistep-transfer contributions, and compute cross sections for selected neutron-rich nuclides. 


\section{Project Management}

\section{Coordination}

- The coordinating P.I. coordinates the different sub-projects, and ensures the cohesion of the overall project.

- Monthly conference calls ensure that practical information is exchanged, and that research projects, visitors and collaborations are properly coordinated.

- Additional conference calls are set up as needed, and our website (see below) is used to deposit internal documents for discussion.

- Collaborative visits and small-group conference calls held on a regular basis to allow for detailed discussions of physics issues.

\section{Website}

We have developed a website at http://www.reactiontheory.org that is hosted at MSU. For the public, this site contains general information about our collaboration, our research papers and talks, the workshops and conferences we attend, and lists of relevant experiments.

For ourselves (protected by a password), we have information about our budget, our plans and deliverables, minutes from our meetings and conference calls, and also a place to deposit internal documents for access by the collaboration.

\section{Collaborative visits in Year 2 Q4 and Year 3 Q1-3}

- Elster went four times to MSU for collaboration meetings, and was accompanied by graduate student Linda Hlophe.

- June 2012: Ch. Elster, J. Escher, and F. Nunes attended HITES 2012 in New Orleans

- June 2012: the whole collaboration met at MSU for annual meeting

- September 2012: the whole collaboration met in Washington, D.C., for the DOE Mid-term review

- November 2012: J. Escher visited OU

- December 2012: Ch. Elster visited LLNL

- February 2013: V. Eremenko visited Ohio University to start the numerical work on the AGS code. 


\section{Postdoctoral Staff and External Collaborators}

\section{TORUS Postdoctoral researcher Dr Neelam Upadhyay}

Year 3 report: Neelam's works this year focused on understanding the generalized AGS formalism introduced by TAMU [26] and exploring the numerical implementation of the momentum-space Coulomb distorted wave representation, particularly for the types of systems we are interested in. In parallel, Neelam also performed additional CDCC calculations to clarify the role of the Coulomb force in $(\mathrm{d}, \mathrm{p})$ reactions involving heavy targets, as well as the sensitivity of the transfer observables to the details of the NN and NA forces. Neelam also finished up some calculations in collaboration with experimentalists at the University of Tennessee. That work was finished early in 2012 and is being written up by other members of the collaboration.

During 2012, Dr. Upadhyay had the opportunity to visit India and give invited seminars at a couple of nuclear physics institutes in Mumbai, as part of her search for the next career move. She has also prepared applications to a number of European funding agencies and awaits the results of the selective processes.

\section{TORUS Postdoctoral researcher Dr Vasily Eremenko}

\section{Ohio University doctoral student Mr Linda Hlophe}

The TORUS grant supported graduate student Linda Hlophe during the Spring quarter 2012, and the Spring semester 2013. During the Summer 2012 and the Fall Semester 2012 he was supported by the DOE contract No. DE-FG02-93ER40756 with Ohio University.

\section{External Visitors in Year 2Q4 and Year 3Q1-3}

- Prof. S.P. Weppner: the grant contributed to two visits (Summer 2012 and Winter 2013), for collaboration on optical potentials.

- A. Deltuva: visited MSU in October 2012

- R. Lazauskas: visited MSU in January 2013

\section{Other Collaborators in Year 2Q4 and Year 3Q1-3}

These collaborations contributed to our project, but were not funded by this grant:

- F.S. Dietrich (LLNL)

- R. Kozub (Tenn. Tech. Univ.)

\section{Planned Visitors in Year 3Q4 and Year 4}

We plan to support the visits of the following people in Year 4 as visitors or consultants:

- Peter C. Tandy (Kent State U., OH) visit to OU

- Steve Weppner (Eckerd College, FL) visit to OU 
- Rimas Lazauskas to visit MSU

- Arnas Deltuva to visit MSU

\section{TORUS Workshops}

\section{Mini-workshop at MSU organized by Nunes}

Organizer F. Nunes

On October 18th 2012, a Mini-workshop on AGS equations and implementation, was held at MSU with Deltuva (Lisbon), Elster (Ohio), Hlope (Ohio), Nunes (MSU), Titus (MSU), and Upadhyay (MSU). Discussions included non-local interactions, separabalizing optical potentials, the momentum-space Coulomb distorted representation and details on the AGS equations as implemented by the Lisbon group.

DNP 2012 Workshop 'And Here Be Dragons: Understanding the Nature of Unstable Isotopes'

\section{J. Escher, I.J. Thompson}

Jutta Escher co-organized (with Calvin Johnson, SDSU) this half-day workshop in the context of the DNP 2012 Fall Meeting. The workshop covered phenomena associated with the effects of the continuum in nuclear physics, including reactions with unstable isotopes. Featured speakers included Ian Thompson and Jolie Cizewski who covered, respectively, theoretical and experimental aspects of $(d, p)$ transfer reactions. Approximately 60 scientists attended. 


\section{Plans for Year 4}

1. We will calculate the matrix elements of the surface operator in the partial wave basis used by DWBA and CDCC calculations, to make a modified Fresco code. This will be tested for transfers to bound states and resonances, supplemented as necessary by interior-post and exterior-prior contributions. The first-order theory of transfers to resonances will be used to analyze existing experiments, and extracted partial widths will be compared to those found by other investigations.

2. Finish the practical implementation of the distorted Coulomb representation (to be used in the AGS equations) and study the properties for a few test cases. Prepare a publication on those results.

3. Find a separable expansion for a suitable neutron-nucleus global potential.

4. Calculate the Coulomb distorted $t$-matrix elements, and refit the separable expansions with a Coulomb term included

5. Begin to implement these separable expansions in existing three-body reaction codes.

6. Implement the AGS equations.

7. We will develop the necessary new semi-microscopic methods to treat doorway states in neutron scattering and capture. Specifically, we will formulate models for pygmy dipole resonances and isobar-analogue resonances, including multistep transfer contributions, to compute capture cross sections for selected neutron-rich nuclides. 


\section{Deliverables}

\subsection{Publications}

1. Published paper [19]: Phys. Rev. C 85, 024613, (2012) - Published February 21, 2012 (omitted in last report).

Utilizing (p,d) and (p,t) reactions to obtain $(n, f)$ cross sections in uranium nuclei via the surrogate-ratio method, R. O. Hughes, C. W. Beausang, T. J. Ross, J. T. Burke, N. D. Scielzo, M. S. Basunia, C. M. Campbell, R. J. Casperson, H. L. Crawford, J. E. Escher, J. Munson, L. W. Phair, and J. J. Ressler.

The surrogate ratio method has been tested for $(\mathrm{p}, \mathrm{d})$ and $(\mathrm{p}, \mathrm{t})$ reactions on uranium nuclei. $236 \mathrm{U}$ and $238 \mathrm{U}$ targets were bombarded with $28-\mathrm{MeV}$ protons and the light ion recoils and fission fragments were detected using the Silicon Telescope Array for Reaction Studies detector array at the 88-Inch Cyclotron at Lawrence Berkeley National Laboratory. The (p,df) reaction channels on 236U and 238U targets were used as a surrogate to determine the $\sigma[236 \mathrm{U}(\mathrm{n}, \mathrm{f})] / \sigma[234 \mathrm{U}(\mathrm{n}, \mathrm{f})]$ cross-section ratio. The (p,tf) reaction channels were also measured with the same targets as a surrogate for the $\sigma[235 \mathrm{U}(\mathrm{n}, \mathrm{f})] / \sigma[(233 \mathrm{U}(\mathrm{n}, \mathrm{f})]$ ratio. For the $(\mathrm{p}, \mathrm{df})$ and $(\mathrm{p}, \mathrm{ff})$ surrogate measurements, there is good agreement with accepted $(n, f)$ values over equivalent neutron energy ranges of $E n=07 \mathrm{MeV}$ and $\mathrm{En}=05.5 \mathrm{MeV}$, respectively. An internal surrogate ratio method comparing the $(\mathrm{p}, \mathrm{d})$ and $(\mathrm{p}, \mathrm{t})$ reaction channels on a single target is also discussed. The $\sigma[234 \mathrm{U}(\mathrm{n}, \mathrm{f})] / \sigma[233 \mathrm{U}(\mathrm{n}, \mathrm{f})]$ and $\sigma[236 \mathrm{U}(\mathrm{n}, \mathrm{f})] / \sigma[235 \mathrm{U}(\mathrm{n}, \mathrm{f})]$ cross-section ratios are extracted using this method for the $236 \mathrm{U}$ and $238 \mathrm{U}$ targets, respectively. The resulting fission cross-section ratios show relatively good agreement with accepted values up to En $<5 \mathrm{MeV}$.

2. Published paper [4]: Phys. Rev. C 85, 044604, (2012) - Published April 2, 2012.

\section{Comparing non-perturbative models of the breakup of neutron-halo nuclei} P. Capel, H. Esbensen and F.M. Nunes.

Breakup reactions of loosely-bound nuclei are often used to extract structure and/or astrophysical information. Here we compare three non-perturbative reaction theories often used when analyzing breakup experiments, namely the continuum discretized coupled channel model, the time-dependent approach relying on a semiclassical approximation, and the dynamical eikonal approximation. Our test case consists of the breakup of ${ }^{15} \mathrm{C}$ on $\mathrm{Pb}$ at $68 \mathrm{MeV} /$ nucleon and $20 \mathrm{MeV} /$ nucleon.

3. Published paper [42]: Phys. Rev. C 85, 054621, (2012) - Published April 23, 2012. Elastic Scattering of ${ }^{6} \mathbf{H e}$ based on a Cluster Description, S. P. Weppner and C. Elster.

Elastic scattering observables (differential cross section and analyzing power) are calculated for the reaction ${ }^{6} \mathrm{He}(\mathrm{p}, \mathrm{p}){ }^{6} \mathrm{He}$ at projectile energies starting at $71 \mathrm{MeV} /$ nucleon. The optical potential needed to describe the reaction is derived describing ${ }^{6} \mathrm{He}$ in terms of a ${ }^{4} \mathrm{He}-$ core and two neutrons. The Watson first order multiple scattering ansatz is extended to accommodate the internal dynamics of a composite cluster model for the ${ }^{6} \mathrm{He}$ nucleus scattering from a nucleon projectile. The calculations are compared with the recent experiments at the projectile energy of $71 \mathrm{MeV} /$ nucleon. In addition, differential cross sections and analyzing powers are calculated at selected higher energies.

4. Published paper [35]: Phys. Rev. Letts. 108, 192701 (2012) - Published May 8, 2012.

\section{Halo nucleus ${ }^{11}$ Be: a spectroscopic study via neutron transfer}

K.T. Schmitt, K.L Jones, A. Bey, S.H. Ahn, D.W. Bardayan, J.C. Blackmon, S. Brown, K.Y. Chae, K.A. Chipps, J.A. Cizewski, K. I. Hahn, J.J. Kolata, R.L. Kozub, J.F. Liang, C. Matei, M. Matos, D. Matyas, B. Moazen, C. Nesaraja, F.M. Nunes, P.D. O’Malley, S.D. Pain, W.A. Peters, S.T. Pittman, A. Roberts, D. Shapira, J.F. Shriner Jr., M.S. Smith, I. Spassova, D.W. Stracener, A.N. Villano and G. Wilson.

The best examples of halo nuclei, exotic systems with a diffuse nuclear cloud surrounding a tightly bound core, are found in the light, neutron-rich region, where the halo neutrons experience only weak binding and a weak, or no, potential barrier. Modern direct-reaction measurement techniques provide powerful probes of the structure of exotic nuclei. Despite more than four decades of these studies on the benchmark one-neutron halo nucleus ${ }^{11} \mathrm{Be}$, the spectroscopic factors for the two bound states remain poorly constrained. In the present work, the ${ }^{10} \mathrm{Be}(\mathrm{d}, \mathrm{p})$ reaction has been used in inverse kinematics at four beam energies to study the structure of ${ }^{11} \mathrm{Be}$. The spectroscopic factors extracted using the adiabatic model were found to be consistent across the four measurements and were largely insensitive to the optical potential used. The extracted spectroscopic factor for a neutron in an $n \ell j=2 s_{1 / 2}$ state coupled to the ground state of ${ }^{10} \mathrm{Be}$ is $0.71(5)$. For the first excited state at $0.32 \mathrm{MeV}$, a spectroscopic factor of $0.62(4)$ is found for the halo neutron in a $1 p_{1 / 2}$ state. 
5. Published paper [33]: Phys. Rev. C 85, 051304(R) (2011) - Published May 24, 2012. Measurement of the entry-spin distribution imparted to the high excitation continuum region of gadolinium nuclei via $(\mathbf{p}, \mathbf{d})$ and $(\mathbf{p}, \mathbf{t})$ reactions

T. J. Ross, C. W. Beausang, R. O. Hughes, J. M. Allmond, C. T. Angell. M. S. Basunia, D. L. Bleuel, J. T. Burke, R. J. Casperson, J. E. Escher, P. Fallon, R. Hatarik, J. Munson, S. Paschalis, M. Petri, L. Phair, J. J. Ressler, N. D. Scielzo, and I. J. Thompson.

Over the last several years, the surrogate reaction technique has been successfully employed to extract $(\mathrm{n}, \mathrm{f})$ and $(\mathrm{n}$, ? ) cross sections in the actinide region to a precision of $95 \%$ and $? 20 \%$, respectively. However, attempts to apply the technique in the rare earth region have shown large (factors of 23) discrepancies between the directly measured $(n, ?)$ and extracted surrogate cross sections. One possible origin of this discrepancy lies in differences between the initial spin-parity population distribution in the neutron induced and surrogate reactions. To address this issue, the angular momentum transfer to the high excitation energy quasicontinuum region in Gd nuclei has been investigated. The (p,d) and $(\mathrm{p}, \mathrm{t})$ reactions on 154,158Gd at a beam energy of $25 \mathrm{MeV}$ were utilized. Assuming a single dominant angular momentum transfer component, the measured angular distribution for the $(\mathrm{p}, \mathrm{d})$ reactions is well reproduced by distorted-wave Born approximation (DWBA) calculations for $\Delta L=4 \hbar$ transfer, whereas the $(\mathrm{p}, \mathrm{t})$ reactions are better characterized by $\Delta L=5 \hbar$. A linear combination of DWBA calculations, weighted according to a distribution of $\mathrm{L}$ transfers (peaking around $\Delta L=4-5 \hbar$ ), is in excellent agreement with the experimental angular distributions.

6. Published paper [39]: Phys. Rev. C 85, 054621 (2011) - Published May 25, 2012. Testing the continuum discretized coupled channel method for deuteron induced reactions, N. J. Upadhyay, A. Deltuva, F. M. Nunes.

The Continuum Discretized Coupled Channels (CDCC) method is a well established theory for direct nuclear reactions which includes breakup to all orders. Alternatively, the 3-body problem can be solved exactly within the Faddeev formalism which explicitly includes breakup and transfer channels to all orders. With the aim to understand how CDCC compares with the exact 3-body Faddeev formulation, we study deuteron induced reactions on: i) ${ }^{10} \mathrm{Be}$ at $E_{\mathrm{d}}=21.4,40.9$ and $71 \mathrm{MeV}$; ii) ${ }^{12} \mathrm{C}$ at $E_{\mathrm{d}}=12$ and $56 \mathrm{MeV}$; and iii) ${ }^{48} \mathrm{Ca}$ at $E_{\mathrm{d}}=56 \mathrm{MeV}$. We calculate elastic, transfer and breakup cross sections. Overall, the discrepancies found for elastic scattering are small with the exception of very backward angles. For transfer cross sections at low energy $\sim 10 \mathrm{MeV} / \mathrm{u}, \mathrm{CDCC}$ is in good agreement with the Faddeev-type results and the discrepancy increases with beam energy. On the contrary, breakup observables obtained with CDCC are in good agreement with Faddeev-type results for all but the lower energies considered here.

7. Published paper [26]: Phys. Rev. C: 860324001 (2012) - Published September 10, 2012 Generalized Faddeev equations in the Alt-Grassberger-Sandhas form for deuteron stripping with explicit inclusion of target excitations and Coulomb interaction,

\section{A.M. Mukhamedzhanov, V. Eremenko and A.I. Sattarov.}

Theoretical description of reactions in general, and the theory for $(d, p)$ reactions, in particular, needs to advance into the new century. Here deuteron stripping processes off a target nucleus consisting of $A$ nucleons are treated within the framework of the few-body integral equations theory. The generalized Faddeev equations in the AGS form, which take into account the target excitations, with realistic optical potentials provide the most advanced and complete description of the deuteron stripping. The main problem in practical application of such equations is the screening of the Coulomb potential, which works only for light nuclei. In this paper we present a new formulation of the Faddeev equations in the AGS form taking into account the target excitations with explicit inclusion of the Coulomb interaction. By projecting the $(A+2)$-body operators onto target states, matrix three-body integral equations are derived which allow for the incorporation of the excited states of the target nucleons. Using the explicit equations for the partial Coulomb scattering wave functions in the momentum space we present the AGS equations in the Coulomb distorted wave representation without screening procedure. We also use the explicit expression for the off-shell two-body Coulomb scattering $T$-matrix which is needed to calculate the effective potentials in the AGS equations. The integrals containing the off-shell Coulomb T-matrix are regularized to make the obtained equations suitable for calculations. For $N N$ and nucleon-target nuclear interactions we assume the separable potentials what significantly simplifies solution of the AGS equations.

8. Published paper[43]: Phys. Rev. C: 86032802 (2012) - Published September 14, 2012 Structures of Exotic ${ }^{131 ; 133}$ Sn Isotopes for r-process nucleosythesis, Shi-Sheng Zhang, M. S. Smith, G. Arbanas, and R. L. Kozub.

Background Four strong single-particle bound levels with strikingly similar level spacings have recently been measured in ${ }^{131} \mathrm{Sn}$ and ${ }^{133} \mathrm{Sn}$. This similarity has not yet been addressed with a theoretical nuclear structure model. Information on these single particle bound levels, as well as on resonant levels above the neutron capture threshold, are also needed to determine neutron capture cross sections and corresponding capture reaction rates - on ${ }^{130 ; 132} \mathrm{Sn}$. The ${ }^{130} \mathrm{Sn}(n, \gamma)$ rate was shown in a recent sensitivity study to significantly impact the synthesis of heavy elements in the r-process in supernovae. Purpose Understand the structure of bound and resonant levels in ${ }^{131 ; 133} \mathrm{Sn}$, and determine if the densities of unbound resonant levels are sufficiently high to warrant statistical model treatments of neutron capture on ${ }^{130 ; 132} \mathrm{Sn}$. Method Single-particle bound and resonant levels for ${ }^{131 ; 133} \mathrm{Sn}$ are self-consistently calculated by the analytical continuation of the coupling constant (ACCC) based on a relativistic mean field (RMF) theory with BCS approximation. Results We 
obtain four strong single-particle bound levels in both ${ }^{131 ; 133} \mathrm{Sn}$ with an ordering that agrees with experiments and spacings that, while differing from experiment, are consistent between the $\mathrm{Sn}$ isotopes. We also find at most one single-particle level in the effective energy range for neutron captures in the r-process. Conclusions Our RMF+ACCC+BCS model successfully reproduces observed single-particle bound levels in ${ }^{131 ; 133} \mathrm{Sn}$ and self-consistently predicts single-particle resonant levels with densities too low for widely used traditional statistical model treatments of neutron capture cross sections on ${ }^{130 ; 132} \mathrm{Sn}$ employing Fermi gas level density formulations.

9. Published paper [27]: Phys. Rev. Lett. 109, 141101 (2012). - Published Oct 1, 2012 Low-temperature triple-alpha rate in a full three-body model N.B. Nguyen, F.M. Nunes, I.J. Thompson and E.F. Brown A

A new three-body method is used to compute the rate of the triple-alpha capture reaction, which is the primary source of ${ }^{12} \mathrm{C}$ in stars In this Letter, we combine the Faddeev hyperspherical harmonics and the R-matrix method to obtain a full solution to the three-body $\alpha+\alpha+\alpha$ continuum. Particular attention is paid to the long-range effects caused by the pairwise Coulomb interactions. The new rate agrees with the Nuclear Astrophysics Compilation of Reaction rates for temperatures greater than 0.07 GK, but a large enhancement at lower temperature is found $\left(\sim 10^{12}\right.$ at $\left.0.02 \mathrm{GK}\right)$. Our results are compared to previous calculations where additional approximations were made. We show that the new rate does not significantly change the evolution of stars around one solar mass. In particular, such stars still undergo a red-giant phase consistent with observations, and no significant differences are found in the final white dwarfs.

10. Published paper [25]: Phys. Rev. C: 86044615 (2012) - Published October 15, 2012 Coulomb renormalization and ratio of proton and neutron asymptotic normalization coefficients for mirror nuclei, A.M. Mukhamedzhanov

Asymptotic normalization coefficients (ANCs) are fundamental nuclear constants playing an important role in nuclear reactions, nuclear structure, and nuclear astrophysics. In this paper the physical reasons for the Coulomb renormalization of the ANC are addressed. Using the Pinkston-Satchler equation the ratio for the proton and neutron ANCs of mirror nuclei is obtained in terms of the Wronskians from the radial overlap functions and regular solutions of the two-body Schrdinger equation with the short-range interaction excluded. This ratio allows one to use microscopic overlap functions for mirror nuclei in the internal region, where they are the most accurate, to correctly predict the ratio of the ANCs for mirror nuclei, which determine the amplitudes of the tails of the overlap functions. Calculations presented for different nuclei demonstrate the Coulomb renormalization effects and independence of the ratio of the nucleon ANCs for mirror nuclei on the channel radius. This ratio is valid both for bound states and resonances. One of the goals of this paper is to draw attention to the possibility of using the Coulomb renormalized ANCs rather than the standard ones, especially when the standard ANCs are too large.

11. Published paper: [20]: Phys. Rev. Lett. 1109172501 (2012) - Published October 24, 2012 Neutron single particle structure in ${ }^{131} \mathrm{Sn}$ and direct neutron capture cross sections R. L. Kozub, G. Arbanas, A. S. Adekola, D. W. Bardayan, J. C. Blackmon, K.Y. Chae, K. A. Chipps, J. A. Cizewski, L. Erikson, R. Hatarik, W. R. Hix, K. L. Jones, W. Krolas, J. F. Liang, Z. Ma, C. Matei, B. H. Moazen, C. D.Nesaraja, S. D. Pain, D. Shapira, J. F. Shriner, Jr., M. S. Smith, and T. P. Swan.

Recent calculations suggest that the rate of neutron capture by $130 \mathrm{Sn}$ has a significant impact on late-time nucleosynthesis in the r-process. Direct capture into low-lying bound states is expected to be significant in neutron capture near the $\mathrm{N}=82$ closed shell, so r-process reaction rates may be strongly impacted by the properties of neutron single particle states in this region. In order to investigate these properties, the $(d, p)$ reaction has been studied in inverse kinematics using a $630 \mathrm{MeV}$ beam of ${ }^{130} \mathrm{Sn}(4.8 \mathrm{MeV} / \mathrm{u})$ and a $\left(\mathrm{CD}_{2}\right)_{n}$ target. An array of Si strip detectors, including SIDAR and an early implementation of the ORRUBA, was used to detect reaction products. Results for the ${ }^{130} \operatorname{Sn}(d, p)^{131} \mathrm{Sn}$ reaction are found to be very similar to those from the previously reported ${ }^{132} \mathrm{Sn}(d, p)^{133} \mathrm{Sn}$ reaction. Directsemidirect $(n, \gamma)$ cross section calculations, based for the first time on experimental data, are presented. The uncertainties in these cross sections are thus reduced by orders of magnitude from previous estimates.

12. Published paper [21]: Phys. Rev. Letts. 109232701 - Published Dec 4, 2012 Measurement of the $-3 \mathrm{keV}$ resonance in the reaction ${ }^{13} \mathbf{C}(\alpha, \mathbf{n})^{16} \mathrm{O}$ of importance in the $s$-process M. La Cognata, C. Spitaleri, O. Trippella, G. G. Kiss, G. V. Rogachev, A. M. Mukhamedzhanov, M. Avila, G. L. Guardo, E. Koshchiy, A. Kuchera, L. Lamia, S. M. R. Puglia, S. Romano, D. Santiago, and R. Spartà,

The ${ }^{13} \mathrm{C}(\alpha, n){ }^{16} \mathrm{O}$ reaction is the neutron source for the main component of the s-process, responsible for the production of most nuclei in the mass range $90<A<204$. It is active inside the helium-burning shell in asymptotic giant branch stars, at temperatures $\lesssim 10^{8}$ $\mathrm{K}$, corresponding to an energy interval where the ${ }^{13} \mathrm{C}(\alpha, \mathrm{n}){ }^{16} \mathrm{O}$ is effective from 140 to $230 \mathrm{keV}$. In this region, the astrophysical $\mathrm{S}(\mathrm{E})$ factor is dominated by the $-3 \mathrm{keV}$ subthreshold resonance due to the $6.356 \mathrm{MeV}$ level in ${ }^{17} \mathrm{O}$, giving rise to a steep increase of the $\mathrm{S}(\mathrm{E})$-factor. Notwithstanding that it plays a crucial role in astrophysics, no direct measurements exist inside the s-process energy window. The magnitude of its contribution is still controversial as extrapolations, e.g., through the $\mathrm{R}$ matrix and indirect techniques, such as the asymptotic normalization coefficient (ANC), yield inconsistent results. The discrepancy amounts to a factor of 3 or more right at 
astrophysical energies. Therefore, we have applied the Trojan horse method to the ${ }^{13} \mathrm{C}\left({ }^{6} \mathrm{Li}, \mathrm{n}^{16} \mathrm{O}\right) \mathrm{d}$ quasifree reaction to achieve an experimental estimate of such contribution. For the first time, the ANC for the $6.356 \mathrm{MeNV}$ level has been deduced through the Trojan horse method as well as the n-partial width, allowing to attain an unprecedented accuracy in the ${ }^{13} \mathrm{C}(\alpha, \mathrm{n}){ }^{16} \mathrm{O}$ study. Though a larger ANC for the $6.356 \mathrm{MeV}$ level is measured, our experimental $\mathrm{S}(\mathrm{E})$-factor agrees with the most recent extrapolation in the literature in the $140-230 \mathrm{keV}$ energy interval, the accuracy being greatly enhanced thanks to this innovative approach.

13. Published paper [30]: J. Phys.: Conf. Ser. 403, 012029 - Published December 18, 2012 Status of reaction theory for studying rare isotopes, F.M. Nunes and N.J. Upadhyay,

Reactions are an important tool to study nuclear structure and for extracting reactions relevant for astrophysics. In this paper we focus on deuteron induced reactions which can provide information on neutron shell evolution as well as neutron capture cross sections. We review recent work on the systematic comparison of the continuum discretized coupled channel method, the adiabatic wave approximation and the Faddeev momentum-space approach. We also explore other aspects of the reaction mechanism and discuss in detail difficulties encountered in the calculations.

14. Published paper [14]: J. Phys.: Conf. Ser. 403012026 - Published December 18, 2012 Exploring R-matrix ideas for the description of one-nucleon transfer reactions, J E Escher, A M Mukhamedzhanov and I J Thompson,

Deuteron-induced reactions, in particular $(\mathrm{d}, \mathrm{p})$ one-neutron transfer reactions, have been used for decades to investigate the structure of nuclei. These reactions, carried out in inverse kinematics, are expected to play a central role in the study of weakly-bound systems at modern radioactive beam facilities. While the theoretical framework and its computational implementation for describing (d,p) reactions have seen much progress over the decades, open questions remain and need to be addressed, including the proper treatment of transfers to resonance states. Recently, a new formalism was proposed [1] that, in principle, describes transfers to both bound and resonance states. The new formalism is summarized here and illustrated; implications are discussed.

15. Published paper [10]: J. Phys.: Conf. Ser. 403, 012025 - Published December 18, 2012 Nuclear Reactions: A Challenge for Few- and Many-Body Theory, Ch. Elster, L. Hlophe,

A current interest in nuclear reactions, specifically with rare isotopes concentrates on their reaction with neutrons, in particular neutron capture. In order to facilitate reactions with neutrons one must use indirect methods using deuterons as beam or target of choice. For adding neutrons, the most common reaction is the $(\mathrm{d}, \mathrm{p})$ reaction, in which the deuteron breaks up and the neutron is captured by the nucleus. Those (d,p) reactions may be viewed as a three-body problem in a many-body context. This contribution reports on a feasibility study for describing phenomenological nucleon-nucleus optical potentials in momentum space in a separable form, so that they may be used for Faddeev calculations of $(\mathrm{d}, \mathrm{p})$ reactions.

16. Published paper [16]: Phys. Rev. C. 87 012801(R) - Published Jan 4, 2013

Suppression of the centrifugal barrier effects in the off-energy-shell neutron $+{ }^{17} \mathrm{O}$ interaction M. Gulino, C. Spitaleri, X. D. Tang, G. L. Guardo, L. Lamia, S. Cherubini, B. Bucher, V. Burjan, M. Couder, P. Davies, R. deBoer, X. Fang, V. Z. Goldberg, Z. Hons, V. Kroha, L. Lamm, M. La Cognata, C. Li, C. Ma, J. Mrazek, A. M. Mukhamedzhanov, M. Notani, S. OBrien, R. G. Pizzone, G. G. Rapisarda, D. Roberson, M. L. Sergi, W. Tan, I. J. Thompson, and M. Wiescher,

The reaction ${ }^{17} \mathrm{O}(\mathrm{n}, \alpha){ }^{14} \mathrm{C}$ was studied at energies from $E_{\text {c.m. }}=0 t o E_{\text {c.m. }} .=350 \mathrm{keV}$ by using the quasifree deuteron breakup in the three-body reaction ${ }^{17} \mathrm{O}+\mathrm{d} \rightarrow \alpha+{ }^{14} \mathrm{C}+\mathrm{p}$, which extended the Trojan Horse indirect method (THM) to neutron-induced reactions. It is found that the ${ }^{18} \mathrm{O}$ excited state at $E^{*}=8.125 \pm 0.002 \mathrm{MeV}$, observed in THM experiments, is absent in the direct measurement because of its high centrifugal barrier. The angular distributions of the populated resonances have been measured by using this method. The results unambiguously indicate the ability of the THM to overcome the centrifugal barrier suppression effect and to pick out the contribution of the bare nuclear interaction.

\subsection{Papers in press}

1. Forthcoming paper [29]: Proceedings CGS14, World Scientific, in press. Are present reaction theories for studying rare isotopes good enough? F. M. Nunes, P. Capel, R.J. Charity, A. Deltuva, W.Dickhoff, H. Esbensen, R.C. Johnson, N.B. Nguyen, N.J. Upadhyay, S.J. Waldecker. 
Rare isotopes are most often studied through nuclear reactions. Nuclear reactions can be used to obtain detailed structure information but also in connection to astrophysics to determine specific capture rates. In order to extract the desired information it is crucial to have a reliable framework that describes the reaction process accurately. A few recent developments for transfer and breakup reactions will be presented. These include recent studies on the reliability of existing theories as well as effort to reduce the ambiguities in the predicted observables.

2. Forthcoming paper [11]: Few-Body Systems 2013, DOI: 10.1007/s00601-013-0644-y

\section{Microscopic Optical Potentials for Helium-6 scattering off Protons,}

Ch. Elster, A. Orazbayev, S.P. Weppner.

The 20th International IUPAP Conference on Few-Body Problems in Physics, August 20-25, 2012, Fukuoka, Japan

The differential cross section and the analyzing power are calculated for elastic scattering of ${ }^{6} \mathrm{He}$ from a proton target using a microscopic folding optical potential, in which the ${ }^{6} \mathrm{He}$ nucleus is described in terms of a ${ }^{4} \mathrm{He}$-core with two additional neutrons in the valence $\mathrm{p}$-shell. In contrast to previous work of that nature, all contributions from the interaction of the valence neutrons with the target protons are taken into account.

\section{The triple-alpha reaction}

F.M. Nunes, N.B. Nguyen and E. Brown,

Proceedings for the International Conference on Nuclei in the Cosmos, Cairns, August 2012, PoS in press (2012).

\section{Reaction theory for studying rare isotopes}

F.M. Nunes, J. Liu, N.B. Nguyen, L. Titus and N.J. Upadhyay,

Proceedings for 13th International Conference on Nuclear Reaction Mechanisms,

Varenna, June 2012, CERN Proceeding Series (2012).

5. Submitted to monograph chapter [37]: '50 Years of Nuclear BCS', Eds. R.A. Broglia and V. Zelevinsky, World Scientific.

Reaction mechanisms of pair transfer, I.J. Thompson

The mechanisms of nuclear transfer reactions are described for the transfer of two nucleons from one nucleus to another. Two-nucleon overlap functions are defined in various coordinate systems, and their transformation coefficients given between coordinate systems. Post and prior couplings are defined for sequential transfer mechanisms, and it is demonstrated that the combination of 'prior-post' couplings avoids non-orthogonality terms, but does not avoid couplings that do not have good zero-range approximations. The simultaneous and sequential mechanisms are demonstrated for the ${ }^{124} \mathrm{Sn}(\mathrm{p}, \mathrm{t})^{122} \mathrm{Sn}$ reaction at $25 \mathrm{MeV}$ using shell-model overlap functions. The interference between the various simultaneous and sequential amplitudes is shown.

\subsection{Presentations}

1. Lectures on FRIB physics, Invited Lectures by Filomena Nunes, Lattice QCD summer school, INT, August 2012

2. Status of reaction theory for studying rare isotopes, Invited Talk by Filomena Nunes, 13th International conference on Nuclear Reactions Mechanisms, Varenna, 11-15 June 2012

3. Status of reaction theory for studying rare isotopes, Invited Talk by Filomena Nunes, HITES, New Orleans, 4-7 June 2012

4. Status of reaction theory for $(d, p)$ reactions, Invited Talk by Filomena Nunes, Colloquium at University of Connecticut, 6th April 2012

5. Reaction theory for exotic nuclei, Invited Seminar by Neelam Upadhyay, Nuclear Physics Division, Bhabha Atomic Research Centre, Mumbai, India May 2012.

6. Reaction theory for exotic nuclei, Invited Seminar by Neelam Upadhyay, Centre for Excellence in Basic Sciences, University of Mumbai, Mumbai, India, May 2012. 
7. Comparing CDCC, Faddeev and Adiabatic models; Talk by Neelam Upadhyay, TORUS Second Year Review, NSCL, Michigan State University, East Lansing, USA, June 2012.

8. INT workshop on 'Structure of Light Nuclei', October 7-12, 2012, Seattle, WA, 'Spin phenomena is elastic scattering of Helium-6 off Protons', Inv. Talk, Ch. Elster

9. 25th Midwest Nuclear Theory Get-Together, Sept. 7-8, 2012, Argonne, IL, 'Towards a Faddeev Description of (d,p) Reactions: Separabilization of Optical Potentials', Ch. Elster, L. Hlophe

10. Horizons of Innovative Theories, Experiments, and Supercomputing in Nuclear Physics (HITES 2012), June 4-7, 2012, New Orleans, LA, 'Nuclear Reactions: A Challenge for Few- and Many Body Theories', Inv. Talk, Ch. Elster, arXiv:1209.0838 [nucl-th], Ch. Elster and L. Hlophe, J. Phys.: Conf. Ser.403 012025 (2012).

11. 11th Conference on the Intersections of Particle and Nuclear Physics (CIPANP) 2012, May 29-June 3, 2012, St Petersburg, FL, 'Theoretical considerations of internal spin for elastic nucleon-nucleus scattering of ${ }^{6} \mathrm{He}$, S.P. Weppner, A. Orazbayev, and Ch. Elster.

12. Annual Meeting of the Ohio Section of APS (OSS12), April 13-14, 2012, Columbus, OH, 'Polarization Phenomena in the Reaction ${ }^{6} \mathrm{He}(\mathrm{p}, \mathrm{p}){ }^{6} \mathrm{He}$, A. Orazbayev, S.P. Weppner, Ch. Elster.

13. Exploring R-matrix ideas for the description of one-nucleon transfer reactions, Jutta Escher, LLNL, May 2012.

14. Using R-matrix ideas to describe one-nucleon transfers to resonance states, Jutta Escher, invited talk at the HITES 2012 conference, New Orleans, June 2012.

15. Status of Reaction Theory for Studying Rare Isotopes, Filomena Nunes, 13th International Conference on Nuclear Reaction Mechanisms, Varenna, June 2012.

16. Exploring R-matrix Ideas for the Description of One-nucleon Transfers to Resonance States, Jutta Escher, DNP 2012 Fall Meeting, Newport Beach, CA, October 2012.

17. Reaction Theory Developments for Nuclear Astrophysics and Other Applications, Jutta Escher, Ohio University, November 2012.

18. Reaction Theory Advances For FRIB, Ian Thompson, invited talk at the DNP 2012 Fall Meeting, Newport Beach, CA, October 2012. 


\section{References}

[1] E. O. Alt, L. D. Blokhintsev, A. M. Mukhamedzhanov, and A. I. Sattarov, Deuteron elastic scattering and stripping processes off C-12 as a three-body problem, Phys. Rev., C75 (2007), p. 054003.

[2] A.M. and Lane, Isobaric spin dependence of the optical potential and quasi-elastic $(p, n)$ reactions, Nuclear Physics, 35 (1962), pp. 676 - 685.

[3] N. Austern, Y. Iseri, M. Kamimura, M. Kawai, G. Rawitscher, and M. Yahiro, Continuum-discretized coupledchannels calculations for three-body models of deuteron-nucleus reactions, Phys. Rep., 154 (1987), pp. 125-204.

[4] P. Capel, H. Esbensen, and F. M. Nunes, Comparing nonperturbative models of the breakup of neutron-halo nuclei, Phys. Rev. C, 85 (2012), p. 044604.

[5] J. A. Cizewski, B. Manning, K. L. Jones, R. L. Kozub, S. Ahn, G. Arbanas, D. W. Bardayan, J. C. Blackmon, K. Y. Chae, K. A. Chipps, S. Hardy, R. Hatarik, M. E. Howard, R. Kapler, J. F. Liang, M. Matos, B. H. Moazen, C. D. Nesaraja, F. M. Nunes, P. D. O’Malley, S. D. Pain, W. A. Peters, S. T. Pittman, A. Ratkiewicz, K. T. Schmitt, D. Shapira, and M. S. Smith, Neutron transfer reactions with tin beams and r-process nucleosynthesis, XII International Symposium on Nuclei in the Cosmos, Cairns, Australia, (2012).

[6] A. Deltuva and A. C. Fonseca, Three-body faddeev-alt-grassberger-sandhas approach to direct nuclear reactions, Phys. Rev. C, 79 (2009), p. 014606.

[7] A. Deltuva, A. C. Fonseca, and P. U. Sauer, Momentum-space treatment of the coulomb interaction in threenucleon reactions with two protons, Phys. Rev. C, 71 (2005), p. 054005.

[8] P. Descouvemont and D. Baye, The R-matrix theory, Reports on Progress in Physics, 73 (2010), p. 036301.

[9] E. I. Dolinskii and A. M. Mukhamedzhanov, Sov. J. Nucl. Phys., 3 (1966), p. 180.

[10] C. Elster and L. Hlophe, Nuclear reactions: A challenge for few- and many-body theory, Journal of Physics: Conference Series, $\mathbf{4 0 3}$ (2012), p. 012025.

[11] C. Elster, A. Orazbayev, and S. Weppner, Microscopic optical potentials for helium-6 scattering off protons, Few-Body Systems, (2013), pp. 1-5.

[12] D. Ernst, J. Londergan, E. Moniz, and R. Thaler, Energy-dependent separable potentials, Phys.Rev., C10 (1974), pp. 1708-1721.

[13] D. Ernst, C. Shakin, and R. Thaler, Separable Representations of Two-Body Interactions, Phys.Rev., C8 (1973), pp. 46-52.

[14] J. E. Escher, A. M. Mukhamedzhanov, and I. J. Thompson, Exploring r-matrix ideas for the description of one-nucleon transfer reactions, Journal of Physics: Conference Series, 403 (2012), p. 012026.

[15] I. M. Gel'fand and G. E. Silov, Generalized Functions and Operations on Them, Fizmatgiz, Moskva, 1958.

[16] M. Gulino, C. Spitaleri, X. D. Tang, G. L. Guardo, L. Lamia, S. Cherubini, B. Bucher, V. Burjan, M. Couder, P. Davies, R. deBoer, X. Fang, V. Z. Goldberg, Z. Hons, V. Kroha, L. Lamm, M. La Cognata, C. Li, C. Ma, J. Mrazek, A. M. Mukhamedzhanov, M. Notani, S. O’Brien, R. G. Pizzone, G. G. Rapisarda, D. Roberson, M. L. Sergi, W. Tan, I. J. Thompson, and M. Wiescher, Suppression of the centrifugal barrier effects in the off-energy-shell neutron $+{ }^{17} \mathrm{O}$ interaction, Phys. Rev. C, 87 (2013), p. 012801.

[17] J. Haidenbauer, Y. Koike, and W. Plessas, Separable representation of the Bonn nucleon-nucleon potential, Phys.Rev., C33 (1986), pp. 439-446.

[18] J. Haidenbauer and W. Plessas, Separable representation of the Paris nucleon nucleon potential, Phys.Rev., C30 (1984), pp. 1822-1839.

[19] R. O. Hughes, C. W. Beausang, T. J. Ross, J. T. Burke, N. D. Scielzo, M. S. Basunia, C. M. Campbell, R. J. Casperson, H. L. Crawford, J. E. Escher, J. Munson, L. W. Phair, and J. J. Ressler, Utilizing (p,d) and (p,t) reactions to obtain $(n, f)$ cross sections in uranium nuclei via the surrogate-ratio method, Phys. Rev. C, 85 (2012), p. 024613.

[20] R. L. Kozub, G. Arbanas, A. S. Adekola, D. W. Bardayan, J. C. Blackmon, K. Y. Chae, K. A. Chipps, J. A. Cizewski, L. Erikson, R. Hatarik, W. R. Hix, K. L. Jones, W. Krolas, J. F. Liang, Z. Ma, C. Matei, B. H. Moazen, C. D. Nesaraja, S. D. Pain, D. Shapira, J. F. Shriner, M. S. Smith, and T. P. Swan, Neutron single particle structure in ${ }^{131}$ Sn and direct neutron capture cross sections, Phys. Rev. Lett., 109 (2012), p. 172501. 
[21] M. La Cognata, C. Spitaleri, O. Trippella, G. G. Kiss, G. V. Rogachev, A. M. Mukhamedzhanov, M. Avila, G. L. Guardo, E. Koshchiy, A. Kuchera, L. Lamia, S. M. R. Puglia, S. Romano, D. Santiago, and R. Spartà, Measurement of the -3 kev resonance in the reaction ${ }^{13} C(\alpha, n){ }^{16} O$ of importance in the s-process, Phys. Rev. Lett., 109 (2012), p. 232701.

[22] A. M. Lane and R. G. Thomas, Rev. Mod. Phys., 30 (1958), p. 257.

[23] K. Miyagawa and Y. Koike, Prog. Theor. Phys., 82 (1989), p. 329.

[24] A. M. Mukhamedzhanov, Theory of deuteron stripping: From surface integrals to a generalized R-matrix approach, Phys. Rev. C, 84 (2011), p. 044616.

[25] A. M. Mukhamedzhanov, Coulomb renormalization and ratio of proton and neutron asymptotic normalization coefficients for mirror nuclei, Phys. Rev. C, 86 (2012), p. 044615.

[26] A. M. Mukhamedzhanov, V. Eremenko, and A. I. Sattarov, Generalized faddeev equations in the alt-grassbergersandhas form for deuteron stripping with explicit inclusion of target excitations and coulomb interaction, Phys. Rev. C, 86 (2012), p. 034001.

[27] N. B. Nguyen, F. M. Nunes, I. J. Thompson, and E. F. Brown, Low-temperature triple-alpha rate in a full threebody nuclear model, Phys. Rev. Lett., 109 (2012), p. 141101.

[28] F. Nunes and N. Upadhyay, Testing three body models, talk presented at the TORUS mid-term review, (Sep 2012).

[29] F. M. Nunes, P. Capel, R. Charity, A. Deltuva, W.Dickhoff, H. Esbensen, R. Johnson, N. Nguyen, N. Upadhyay, and S. Waldecker, Are present reaction theories for studying rare isotopes good enough?, Proceedings CGS14, World Scientific, in press., (2012).

[30] F. M. Nunes and N. J. Upadhyay, Status of reaction theory for studying rare isotopes, Journal of Physics: Conference Series, 403 (2012), p. 012029.

[31] E. Radermacher, M. Wilhelm, S. Albers, J. Eberth, N. Nicolay, H. Thomas, H. Tiesler, P. von Brentano, R. Schwengner, S. Skoda, G. Winter, and K. Maier, The $\gamma$-decay of particle-hole states in ${ }^{208} \mathrm{~Pb}$ using the Euroball Cluster detector, Nuclear Physics A, 597 (1996), pp. 408 - 426.

[32] R. Richardson and N. Sherman, Exact eigenstates of the pairing-force hamiltonian, Nuclear Physics, 52 (1964), pp. 221, 253.

[33] T. J. Ross, C. W. Beausang, R. O. Hughes, J. M. Allmond, C. T. Angell, M. S. Basunia, D. L. Bleuel, J. T. Burke, R. J. Casperson, J. E. Escher, P. Fallon, R. Hatarik, J. Munson, S. Paschalis, M. Petri, L. Phair, J. J. Ressler, N. D. Scielzo, and I. J. Thompson, Measurement of the entry-spin distribution imparted to the high excitation continuum region of gadolinium nuclei via $(p, d)$ and $(p, t)$ reactions, Phys. Rev. C, 85 (2012), p. 051304.

[34] T. J. Ross, C. W. Beausang, R. O. Hughes, N. D. Scielzo, J. T. Burke, J. M. Allmond, C. T. Angell, M. S. Basunia, D. L. Bleuel, R. J. Casperson, J. E. Escher, P. Fallon, R. Hatarik, J. Munson, S. Paschalis, M. Petri, L. Phair, and J. J. Ressler, Spectroscopy of ${ }^{88} Y$ by the $(p, d \gamma)$ reaction, Phys. Rev. C, 86 (2012), p. 067301.

[35] K. T. Schmitt, K. L. Jones, A. Bey, S. H. Ahn, D. W. Bardayan, J. C. Blackmon, S. M. Brown, K. Y. Chae, K. A. Chipps, J. A. Cizewski, K. I. Hahn, J. J. Kolata, R. L. Kozub, J. F. Liang, C. Matei, M. Matoš, D. Matyas, B. Moazen, C. Nesaraja, F. M. Nunes, P. D. O’Malley, S. D. Pain, W. A. Peters, S. T. Pittman, A. Roberts, D. Shapira, J. F. Shriner, M. S. Smith, I. Spassova, D. W. Stracener, A. N. Villano, and G. L. Wilson, Halo nucleus ${ }^{11}$ Be: A spectroscopic study via neutron transfer, Phys. Rev. Lett., 108 (2012), p. 192701.

[36] B. B. Skorodumov, G. V. Rogachev, A. Aprahamian, V. Z. Goldberg, J. J. Kolata, S. Almaraz, H. Amro, E. D. Johnson, L. O. Lamm, M. Quinn, A. Teymurazyan, and A. Woehr, $T=3 / 2$ states in ${ }^{13} \mathrm{C}$, Phys. Rev. C, 78 (2008), p. 044603.

[37] I. J. Thompson, Reaction mechanisms of pair transfer, (2012).

[38] N. Upadhyay, Report on coulomb distorted effective potentials, MSU report, (Feb 2013).

[39] N. J. Upadhyay, A. Deltuva, and F. M. Nunes, Testing the continuum-discretized coupled channels method for deuteron-induced reactions, Phys. Rev. C, 85 (2012), p. 054621.

[40] R. Varner, W. Thompson, T. McAbee, E. Ludwig, and T. Clegg, A global nucleon optical model potential, Phys.Rept., 201 (1991), pp. 57-119.

[41] S. Weppner, R. Penney, G. Diffendale, and G. Vittorini, An Isospin dependent global nucleon-nucleus optical model at intermediate energies, Phys.Rev., C80 (2009), p. 034608.

[42] S. P. Weppner and C. Elster, Elastic scattering of ${ }^{6}$ he based on a cluster description, Phys. Rev. C, 85 (2012), p. 044617.

[43] S.-S. Zhang, M. S. Smith, G. Arbanas, and R. L. Kozub, Structures of exotic ${ }^{131,133}$ sn isotopes and effect on r-process nucleosynthesis, Phys. Rev. C, 86 (2012), p. 032802. 